\title{
Enhanced Abyssal Mixing in the Equatorial Pacific Associated with Non-Traditional Effects
}

\author{
Bertrand L. Delorme, ${ }^{a}$ Leif N. Thomas, ${ }^{a}$ Patrick Marchesiello,,${ }^{b}$ Jonathan Gula,,${ }^{c, d}$ \\ Guillaume Roullet, ${ }^{\mathrm{c}}$ AND M. Jeroen Molemaker ${ }^{\mathrm{e}}$ \\ ${ }^{a}$ Earth System Science Department, Stanford University, Stanford, California \\ ${ }^{\mathrm{b}}$ IRD/LEGOS, Toulouse, France \\ ${ }^{\mathrm{c}}$ Univ. Brest, CNRS, IRD, Ifremer, Laboratoire d'Océanographie Physique et Spatiale, IUEM, Brest, France \\ ${ }^{\mathrm{d}}$ Institut Universitaire de France, Paris, France \\ ${ }^{\mathrm{e}}$ Department of Atmospheric and Oceanic Sciences, University of California, Los Angeles, Los Angeles, California
}

(Manuscript received 3 October 2020, in final form 11 March 2021)

\begin{abstract}
Recent theoretical work has shown that, when the so-called nontraditional effects are taken into account, the reflection of equatorially trapped waves (ETWs) off the seafloor generates strong vertical shear that results in bottomintensified mixing at the inertial latitude of the ETW via a mechanism of critical reflection. It has been estimated that this process could play an important role in driving diapycnal upwelling in the abyssal meridional overturning circulation (AMOC). However, these results were derived under an idealized configuration with a monochromatic ETW propagating through a flat ocean at rest. To test the theory in a flow that is more representative of the ocean, we contrast a set of realistic numerical simulations of the eastern equatorial Pacific run using either the hydrostatic or quasi-hydrostatic approximation, the latter of which accounts for nontraditional effects. The simulations are nested into a Pacific-wide hydrostatic parent solution forced with climatological data and realistic bathymetry, resulting in an ETW field and a deep circulation consistent with observations. Using these simulations, we observe enhanced abyssal mixing in the quasi-hydrostatic run, even over smooth topography, that is absent in the hydrostatic run. The mixing is associated with inertial shear that has spatiotemporal properties consistent with the critical reflection mechanism. The enhanced mixing results in a weakening of the abyssal stratification and drives diapycnal upwelling in our simulation, in agreement with the predictions from the idealized simulations. The diapycnal upwelling is $O(10) \mathrm{Sv}\left(1 \mathrm{~Sv} \equiv 10^{6} \mathrm{~m}^{3} \mathrm{~s}^{-1}\right)$ and thus could play an important role in closing the AMOC.
\end{abstract}

KEYWORDS: Tropics; Abyssal circulation; Diapycnal mixing; Waves, oceanic

\section{Introduction}

The abyssal meridional overturning circulation (AMOC) is associated with production of abyssal waters through deep convection around Antarctica. Consequently, mixing processes are required to lift the abyssal waters back up and eventually close the AMOC. Although the polar source regions of abyssal waters have been identified, the energy sources driving mixing and their spatiotemporal distribution are still a subject of debate (Ferrari 2014). More than a decade ago, inverse calculations from Lumpkin and Speer (2007) showed that much of the zonally integrated diapycnal upwelling that closes the AMOC occurs in the tropical oceans, suggesting that intense mixing takes place in these regions. However, we lack both observational evidence and robust theories that could support the inferences from these inverse models.

In a recent paper, Delorme and Thomas (2019, hereafter DT19) showed that surface-generated equatorially trapped waves (ETWs) can energize mixing in the abyss of the tropical oceans upon reflection off the seafloor when the full Coriolis force and so-called nontraditional (NT) effects are taken into account. DT19 used an idealized model configuration where the horizontal component of Earth's rotation $\tilde{f}$ is nonzero, allowing for NT effects to be triggered. Their domain consists of

Corresponding author: Bertrand Delorme, bdelorme@ stanford.edu an equatorial channel with a flat bottom and periodic zonal boundary conditions. At the surface, monochromatic downwardpropagating ETWs are generated in a 1-km-thick sponge layer and propagate freely in the deep within an ocean at rest. Once they reach the weakly stratified abyss, all types of ETWs-from inertia-gravity waves (IGWs) to Rossby waves - are modified by the NT effects, and they ultimately undergo critical reflection at their inertial latitude where their frequency equals the local inertial frequency $f$. The critical reflection mechanism is triggered by a change in the meridional scale of the reflected waves: NT effects act to break the vertical symmetry of downward-propagating ETWs, and, by so doing, they yield a more complex bottom reflection that requires higher meridional modes to satisfy the boundary conditions. These higher meridional modes are governed by a two-dimensional wave equation for IGWs with NT terms that predicts the occurrence of critical reflection at the inertial latitude of the wave (Gerkema and Shrira 2005; Winters et al. 2011). This process acts to locally enhance the shear, which drives mixing in DT19 simulations. Although the mixing is confined to the inertial latitude, DT19 estimated that it could drive large-scale diapycnal upwelling to the order of $10 \mathrm{~Sv}\left(1 \mathrm{~Sv} \equiv 10^{6} \mathrm{~m}^{3} \mathrm{~s}^{-1}\right)$ and thus significantly contribute to closing the AMOC.

However, DT19 prediction was derived from a highly idealized configuration that has five potential limitations. First, DT19 only considered single monochromatic waves, whereas the equatorial ocean consists of a spectrum of waves. Second, 
the forcing mechanism in DT19 is zonally symmetric and persistent in time, yielding persistent and zonally uniform mixing. In the real ocean, downward-propagating ETWs are generated at the surface through winds (Kessler and McCreary 1993; Kelly et al. 1995; Durland and Farrar 2012) and through upper-ocean instabilities associated with western boundary currents, tropical instability waves (TIWs), or the equatorial undercurrent (Kindle and Thompson 1989; Woodberry et al. 1989; Ascani et al. 2010). Such forcing mechanisms exhibit zonal and meridional variations as well as temporal variability, which ultimately manifest in the ETW field. For example, TIWs occur predominantly on the eastern side of the ocean basin with most of their energy confined primarily north of the equator (Malardé et al. 1987), and they occur mostly between August and December (Qiao and Weisberg 1995). Irregular forcing patterns might lead to areas or periods where most of the waves' energy converges at depth, but also to "shadow zones" where the waves' energy is very weak or episodic. Third, the stratification in DT19 model is horizontally uniform. Horizontal variations in the stratification in the real ocean might have a strong influence on DT19 theory at depth since NT effects are strongly dependent on the value of the bottom stratification. Fourth, the wave in DT19 model propagates in an ocean at rest, whereas the equatorial ocean is known for having a very energetic circulation at depth with the presence of the so-called equatorial deep jets (Cravatte et al. 2017; Ménesguen et al. 2019). Fifth and last, the bathymetry in DT19 configuration is flat. The nonflat bathymetry in the real ocean can affect the occurrence and intensity of critical reflection, an effect that also needs to be investigated.

In this study, our primary goal is to address these potential limitations by investigating how DT19 theory may manifest itself in the real ocean. We would like to know where and when critical reflection due to NT effects should be expected and what its effects are on abyssal mixing and stratification in the equatorial oceans. To answer these questions, we focus on the eastern equatorial Pacific Ocean, using a set of two realistic, high-resolution simulations with and without $\tilde{f}$. These simulations are driven by climatological forcing and use a grid nesting approach from a parent, basin-wide simulation of the Pacific Ocean. A comparison of the simulations with and without $\tilde{f}$ allows us to investigate the influence of NT effects on the properties of the abyssal equatorial ocean.

The remainder of this paper is organized as follows. The numerical model and simulation setup are described in section 2. A comparison of the simulations with and without $\tilde{f}$ is presented in section 3 to highlight the key differences that we observe in the abyssal ocean with NT effects. These differences are interpreted with the theory for critical reflection that has been predicted by DT19 in section 4, and the implications for diapycnal transport in the abyss are discussed. The article is concluded in section 5 with a discussion of the strengths and limitations of the theory and potential topics for future research.

\section{Model setup}

In this section, we introduce the realistic simulations of the Pacific Ocean run with two regional models based on the
Regional Oceanic Modeling System (ROMS; Shchepetkin and McWilliams 2005, 2009b). ROMS solves the primitive equations for the velocity, potential temperature, and salinity, using a full equation of state for seawater (Shchepetkin and McWilliams 2009a, 2011).

We used a one-way nesting procedure to go from a parent grid covering most of the Pacific Ocean to a child grid centered around the eastern equatorial Pacific. The two successive domains are highlighted in Fig. 1a, which shows a snapshot of the sea surface temperature (SST) from the model. One-way nesting has been used with success in the past to obtain local solutions at high resolution while preserving the regional-scale circulation (Penven et al. 2006; Mason et al. 2010; Molemaker et al. 2015; Gula et al. 2015). In this configuration, there is no feedback from the child grid to the parent grid: the parent simulation supplies only boundary and initial conditions to the child simulation offline, and each simulation is run independently. The boundary condition algorithm that we used consists of a modified Flather-type scheme for the barotropic mode (Mason et al. 2010) and an Orlanski-type scheme for the baroclinic mode (Marchesiello et al. 2001).

In our study, the parent simulation was run with the University of California, Los Angeles (UCLA), version of the ROMS model, while the child simulation was run with the Coastal and Regional Ocean Community model (CROCO) version of the ROMS model [see Jullien et al. (2019, section 16.3) for a thorough description of the different versions of ROMS and their historical development]. We used the CROCO version because it has been recently enhanced with the addition of a quasi-hydrostatic $(\mathrm{QH})$ option. The $\mathrm{QH}$ equations do not include the vertical acceleration but do take into account the NT terms [i.e., $\tilde{f} w$ in the zonal momentum equation and $\tilde{f} u$ in the vertical momentum equation, the latter of which modifies the hydrostatic pressure field, and where $(u$, $v, w)$ is the velocity vector]. The appendix describes the $\mathrm{QH}$ implementation of CROCO in more detail, as well as the motivation for developing it. Apart from the QH capabilities, the two versions of ROMS are nearly identical for the purposes of the work presented here.

The parent simulation was constructed similarly to the one introduced in Lemarié et al. (2012) but with a much finer horizontal resolution. It was used previously in Molemaker et al. (2015). It has an orthogonal grid based on a transverse Mercator projection with $1842 \times 962$ points, allowing a smooth transition of the grid size from $12.5 \mathrm{~km}$ at the equator to $8.5 \mathrm{~km}$ at high latitudes. The south and north extremes of the grid are around $40^{\circ} \mathrm{S}$ and $55^{\circ} \mathrm{N}$, respectively. The simulation is run for approximately 10.5 years.

We used the last 6 years of the parent solution at 5-day intervals to force the open boundary conditions of the child grid. This grid covers most of the eastern equatorial Pacific region, from $165^{\circ}$ to $105^{\circ} \mathrm{W}$ and between $\pm 19^{\circ} \mathrm{N}$. Its location was motivated by two criteria: 1) the presence of TIWs at the surface, which flux energy downward in the water column (Ascani et al. 2010), and 2) a smooth bathymetry over a large part of the domain that limits other near-bottom processes that can generate turbulence such as lee waves (Fig. 1b). The child domain has $1100 \times 700$ grid points with a horizontal resolution of $6 \mathrm{~km}$. 

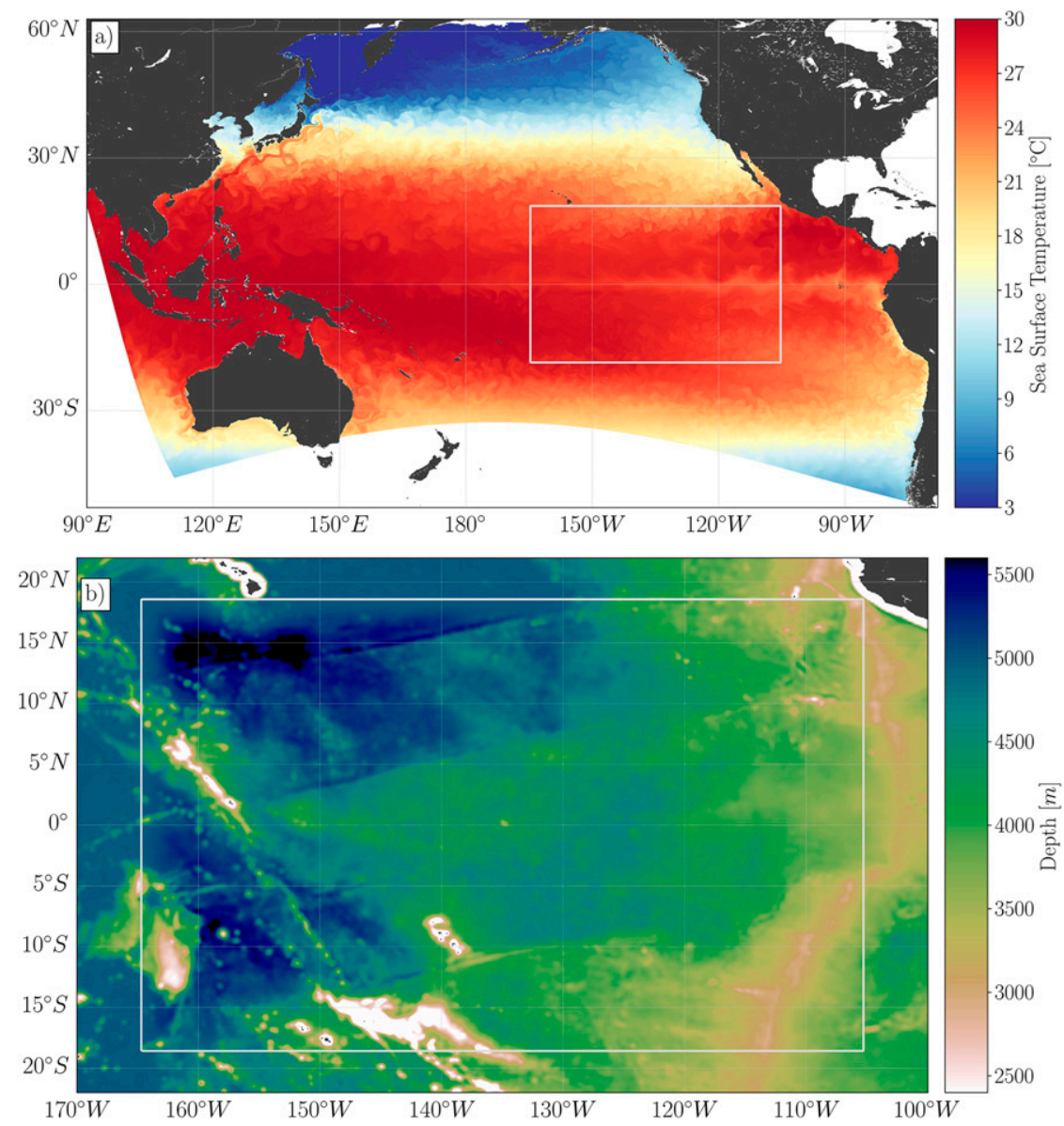

FIG. 1. (a) Instantaneous sea surface temperature in the Pacific basin at the beginning of December as simulated by ROMS. The parent domain covers most of the Pacific Ocean while the child domain is delineated by the thick gray lines (between $165^{\circ}$ and $105^{\circ} \mathrm{W}$ and $\pm 19^{\circ} \mathrm{N}$ ). The SST plotted inside each of the domains is computed using data at their corresponding resolutions. In the eastern part of the basin, the surface signature of TIWs is clearly visible. (b) Seafloor bathymetry in the eastern equatorial Pacific taken from the SRTM30_PLUS dataset and used for the simulations. The bathymetry over most of the child domain is relatively smooth.

We ran the child simulation in both the $\mathrm{QH}$ and hydrostatic $(\mathrm{H})$ mode.

For all simulations, the model bathymetry was interpolated from the Shuttle Radar Topography Mission (SRTM30_ PLUS) dataset based on the 1-min Smith and Sandwell (1997) global dataset, but higher-resolution data were used where available (Becker et al. 2009). The preparation of the topography in the model was split into two successive steps for each simulation. First, a coarsening procedure with a Gaussian smoothing kernel with a width 8 times that of the topographic grid spacing was used to ensure that all the grid metrics are smooth at the scale of the resolution. Second, a so-called logsmoothing procedure was used to ensure that the depth difference between grid cells normalized by the depth $(\Delta H / H)$ did not exceed 0.2 . This criterion is based on computational restrictions for models formulated with a terrain-following coordinate, such as ROMS (see Beckmann and Haidvogel 1993).
To avoid a discontinuity in the grids between the child and parent simulations, the topography near the boundaries of the nested domain was smoothly matched with the parent topography over 32 grid points. The maximum depth for the child grid was set to $6000 \mathrm{~m}$.

The same climatological datasets were used for forcing the lateral boundaries of the parent simulation and the surface forcing for all simulations. At the surface, the wind stress was prepared following a two-step procedure that is described in detail in Lemarié et al. (2012). First, the monthly climatology of QuikSCAT-based Scatterometer Climatology of Ocean Wind (SCOW; Risien and Chelton 2008) was adjusted toward the Tropical Atmosphere Ocean (TAO) buoy data that are available in the equatorial Pacific to prevent a recurring bias that can occur in modeled equatorial currents with SCOW forcing. Then, daily winds from the CERSAT (French ERS Processing and Archiving Facility) Mean Wind Fields (MWF) 
blended product were added to the final wind stress. We obtained heat fluxes from the Common Ocean-Ice Reference Experiment monthly climatology (CORE; Large and Yeager 2009), and incorporated a weak feedback with the modeled SST (Barnier et al. 1995). Surface evaporation and precipitation were taken from the Hamburg Ocean Atmosphere Parameters and Fluxes from Satellite dataset (HOAPS-38; Andersson et al. 2010) with an additional restoring tendency toward climatological monthly surface salinity from the World Ocean Atlas (WOA; Conkright et al. 2002) to prevent surface salinity from drifting away from climatological values. Initial and boundary conditions for temperature, salinity, surface elevation, and the horizontal velocity for the parent domain were taken from the monthly averaged Simple Ocean Data Assimilation (SODA) ocean climatology (Carton and Giese 2008).

In the vertical, the parent simulation has 50 terrain-following $\sigma$ levels that are stretched toward the surface, while the child simulation has 80 vertical levels that are stretched toward both the surface and the bottom. The formulation for the vertical coordinate is described in Shchepetkin and McWilliams [2009a, their Eqs. (2.2) and (2.4)]. In this work, $h_{c}=250 \mathrm{~m}$ for both the parent and the child simulations $\left(h_{c}\right.$ represents the depth of the transition between flat $z$ levels and terrain-following $\sigma$ levels). The parameter controlling the bottom refinement of the grid is $\theta_{b}=1.5$ in the parent simulation and $\theta_{b}=4.5$ in the child simulation. At the surface, we used $\theta_{s}=6.5$ for both simulations.

Since the objective of this paper is to investigate where and when NT effects may occur in the equatorial Pacific, a free-slip boundary condition was used at the bottom with no bottom drag, allowing us to avoid the generation of high shear through friction. This makes the generation of shear through the critical reflection of ETWs modified by NT effects more distinguishable. However, we have also run simulations with bottom drag in which NT effects are evident. These will be discussed more fully in section 4 .

Vertical mixing of tracers and momentum was accomplished using the $K$-profile parameterization (KPP; Large et al. 1994). This parameterization has both boundary layer and interior mixing schemes as described in Large et al. (1994). The interior mixing scheme estimates the mixing coefficients by combining the effects of three mechanisms: shear instability, convective instability and internal wave breaking. Internal wave generated mixing serves to induce a background diffusivity, which was set to the default value of $1 \times 10^{-4} \mathrm{~m}^{2} \mathrm{~s}^{-1}$ for momentum and $1 \times 10^{-5} \mathrm{~m}^{2} \mathrm{~s}^{-1}$ for tracers. The convective component used values of $0.1 \mathrm{~m}^{2} \mathrm{~s}^{-1}$ for both diffusivity and viscosity for unresolved convective cells when $N^{2}<0$, where $N$ is the Brunt-Väisälä frequency. When $N^{2}>0$, interior diffusivities and viscosities due to shear instability were calculated using a gradient Richardson number formulation as

$$
\nu_{s}=\left\{\begin{array}{l}
\nu_{0} \quad \text { if } \quad \mathrm{Ri}<0, \\
\nu_{0}\left[1-\left(\mathrm{Ri}^{2} \mathrm{Ri}^{c}\right)^{2}\right]^{3} \quad \text { if } \quad 0<\mathrm{Ri}<\mathrm{Ri}^{c} \\
0 \quad \text { if } \quad \mathrm{Ri}^{c}<\mathrm{Ri},
\end{array}\right.
$$

where $\nu_{0}=5 \times 10^{-3} \mathrm{~m}^{2} \mathrm{~s}^{-1}$ was the maximum interior mixing coefficient due to shear instability. Ri is the gradient Richardson number defined as

$$
\mathrm{Ri}=N^{2} /\left[\left(\frac{\partial u}{\partial z}\right)^{2}+\left(\frac{\partial v}{\partial z}\right)^{2}\right]
$$

The value of the critical gradient Richardson number below which turbulent mixing occurs, $\mathrm{Ri}^{c}$, was set to the default value of 0.7, as suggested in Large et al. (1994).

Analyses of the abyssal stratification in the parent simulation showed that its values were unrealistically larger than what has been observed in the equatorial Pacific. DT19 showed that weak stratification of the water column is a crucial criterion for NT effects to be effective, that is, the key nondimensional parameter controlling the strength of the NT effects is

$$
\Gamma=N^{2} / \widetilde{f^{2}}
$$

When $\Gamma=O(1)$ or smaller, NT effects are important (see DT19). To have a realistic stratification in the abyss, we modified the stratification profile in the initial and boundary conditions of the child simulation to make it consistent with the observations. To do this, we modified the temperature $T$ and salinity $S$ fields at each horizontal location following

$$
\begin{aligned}
& T_{i j}\left(z_{i j}\right)=T_{i j}\left(z_{i j}\right)\left[1-\mathscr{C l}_{i j}\left(z_{i j}\right)\right]+T_{i j}\left(H_{i j}\right) \mathscr{C l}_{i j}\left(z_{i j}\right), \\
& S_{i j}\left(z_{i j}\right)=S_{i j}\left(z_{i j}\right)\left[1-\mathscr{C l}_{i j}\left(z_{i j}\right)\right]+S_{i j}\left(H_{i j}\right) \mathscr{C l}_{i j}\left(z_{i j}\right),
\end{aligned}
$$

where $i$ and $j$ denote the horizontal indices of the grid in the initial and boundary conditions, $z_{i j}$ denotes the vertical coordinate at location $(i, j), H_{i j}$ is the depth of the water column at location $(i, j)$, and $\mathscr{C}$ is a mask that modulates the correction. The mask $\mathscr{C}$ consists of a hyperbolic tangent function that is zero in the upper water column and approaches one smoothly in the abyssal ocean,

$$
\mathscr{L}_{i j}=\frac{1}{2}\left\{1-\tanh \left[\frac{z_{i j}-\left(H_{i j}+h\right)}{100}\right]\right\},
$$

where $h$ denotes the width of the bottom boundary layer where the stratification is reduced. In the simulations, we set $h=$ $300 \mathrm{~m}$ based on observed stratification profiles. The first year of simulation for the child runs was used as a spinup to allow the system to adjust to these modifications, and we used the remaining 5 years of simulation for the analyses presented in the next sections. An example of the stratification profile in both the parent and child simulations at $110^{\circ} \mathrm{W}$ between $\pm 2^{\circ} \mathrm{N}$, along with profiles from observations, illustrates that $N^{2}$ in the child simulation reaches values that are closer to $\tilde{f}^{2}$ at this location compared to the parent simulation, more in line with the observations (Fig. 2).

\section{Results}

\section{a. Equatorial dynamics}

A comprehensive model validation of the circulation is beyond the scope of this paper, which is devoted to the NT effects. Lemarié et al. (2012) has already conducted a detailed validation of a simulation similar to the parent simulation that we used for this work [our parent simulation has a higher spatial resolution than the one from Lemarié et al. (2012), but 


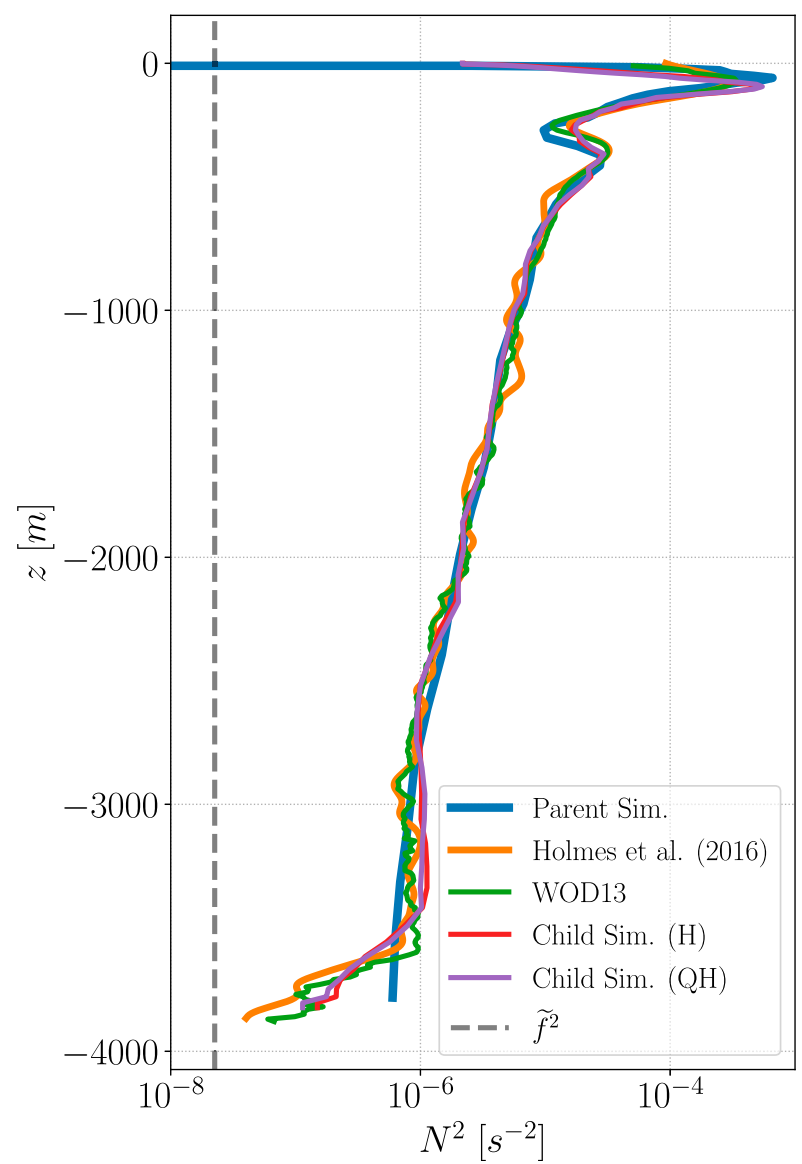

FIG. 2. Meridionally averaged profiles of the stratification $N^{2}$ at $110^{\circ} \mathrm{W}$ between $\pm 2^{\circ}$ measured by Holmes et al. (2016) (orange line) and taken from the parent simulation (blue line) and the child simulations after a 1-yr spinup (the QH case is shown in purple, and the $\mathrm{H}$ case is shown in red). The stratification from all the independent casts available between $109^{\circ}$ and $111^{\circ} \mathrm{W}$ and $\pm 2^{\circ}$ in the World Ocean Database 2013 is also shown (green line). The black dotted line represents the value of $\tilde{f}^{2}$ at the equator. As shown in DT19, NT effects are expected to be stronger when $\Gamma=N^{2} / \tilde{f}^{2}=O(1)$ or lower.

it uses the same numerical algorithms, bathymetry, surface forcing, and boundary conditions]. They showed that the model reproduces the key features of the observed circulation in the equatorial Pacific. In this section, we instead focus on the extent to which NT effects have an effect on the properties of the equatorial Pacific that are the most relevant to the problem at hand, namely, the structure of the mean circulation and the ETW field.

\section{1) MEAN CIRCULATION}

We contrast the mean zonal currents averaged over $120^{\circ}$ $160^{\circ} \mathrm{W}$ in the $\mathrm{H}$ and $\mathrm{QH}$ simulation (Fig. 3). The mean equatorial current system is composed of the eastward flowing North Equatorial Countercurrent, the Equatorial Undercurrent, the South and North Subsurface Countercurrent, the westwardflowing South and North Equatorial Currents, and the
Equatorial Intermediate Current. All of these currents are found in both the $\mathrm{H}$ and $\mathrm{QH}$ simulations with similar spatial structures and amplitudes, and are consistent with observations [see Cravatte et al. (2017) for a similar calculation from the Argo float dataset]. The low-latitude subsurface countercurrents highlighted in Ménesguen et al. (2019) are also visible in both simulations on each side of the equator.

\section{2) EQUATORIAL WAVE FIELD}

Farrar (2008) derived the wavenumber-frequency power spectrum of sea surface height from altimetry data between $7^{\circ} \mathrm{S}$ and $7^{\circ} \mathrm{N}$ to examine the dispersion relation of wavelike variability with periods of about 20-200 days in the equatorial Pacific Ocean (see his Fig. 2). Following up on these results, Farrar and Durland (2012) used the surface dynamic height relative to $500 \mathrm{dbar}$ from the TAO/TRITON moorings between $5^{\circ} \mathrm{S}$ and $5^{\circ} \mathrm{N}$ to derive a wavenumber-frequency power spectrum focusing on wavelike variability with periods of about 3-15 days in the equatorial Pacific Ocean (see their Fig. 5). These two studies provided a quantification of the full ETW spectrum from observations in the equatorial Pacific Ocean. Using a similar approach, we calculated the wavenumber-frequency power spectrum of the surface meridional velocity field averaged over a range of latitudes and longitudes corresponding to the limits of the domain of the child simulations (Fig. 4). In the equatorial regions, meridional velocities are a good proxy for ETWs since the zonal velocity is dominated by the strong zonal flows. However, using the meridional velocity inherently removes the Kelvin wave part of the spectrum from our analysis. Given our model's spatial and temporal resolution, we were able to resolve wavelike variability with periods from 2 days to a year, and zonal wavelengths exceeding $20 \mathrm{~km}$.

The power spectral density of the meridional velocity in the $\mathrm{H}$ simulation is very similar to that in the $\mathrm{QH}$ simulation. It has spectral peaks that lie at similar locations on the analytical dispersion curves of the first and second baroclinic modes in a resting ocean (white lines in Fig. 3), showing that NT effects do not affect the dispersion of the waves as described in DT19. In addition, the spectral amplitude of each wave is also very similar between the $\mathrm{H}$ and $\mathrm{QH}$ simulations. Note that because of the Doppler shift induced by the mean zonal currents on the waves, some maxima in the power spectrum may lie close to but not entirely on the theoretical curves (McPhaden and Knox 1979).

Overall, the model's spectra have similar spectral peaks as the ones calculated by Farrar (2008) and Farrar and Durland (2012) from observations (excluding the Kelvin wave part of the spectrum). The highest peak occurs near 33 days and is associated with the first baroclinic mode Rossby wave. The second largest peak occurs near 17 days and is associated with the westward-propagating Yanai wave. Both of these waves have also been seen in subsurface observations (Lyman et al. 2007), and are thought to be triggered by the TIWs that are clearly visible in the SST field in Fig. 1. At lower frequencies, there is significant energy around 60 days that corresponds to the second baroclinic mode Rossby wave. The Yanai wave dispersion curves for the first and second baroclinic modes 

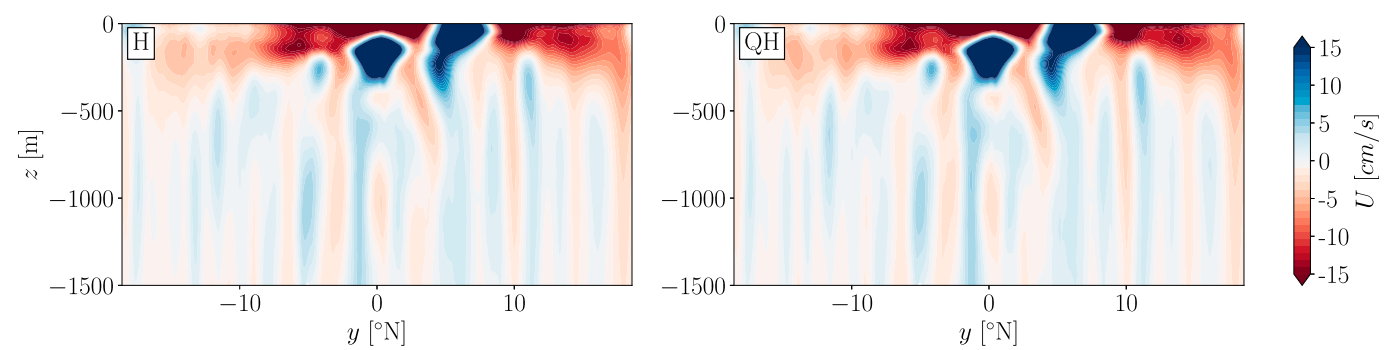

FIG. 3. Mean zonal currents averaged between $120^{\circ}$ and $160^{\circ} \mathrm{W}(U)$ in the (left) $\mathrm{H}$ and (right) QH simulations.

The averaged has been taken over the last 5 years of simulation.

have other peaks at 23,14 , and 10 days that have also been reported in observations (Farrar and Durland 2012; Shinoda 2012). At higher frequencies, we see many elevated spectral peaks at low zonal wavenumbers. The peak around 6 days has also been found by Farrar and Durland (2012), who associated it to the second baroclinic mode of an eastward-propagating IGW. The elevated signal around 5 days was observed by Wunsch and Gill (1976), and is associated with the variability of the meridional winds. According to Farrar and Durland (2012), it would correspond to a double resonance of both the first baroclinic mode, first meridional mode IGW and the second baroclinic mode, second meridional mode IGW.

The model allows us to investigate the ETW spectrum near the bottom as well. Using the same approach as in the previous paragraph, we calculated the wavenumber-frequency power spectrum of the meridional velocity field averaged over the bottom $\sigma$ layer in the child simulations. The results are shown in Fig. 5 for the $\mathrm{H}$ simulation but, as with the surface calculation, they are qualitatively similar to those from the QH run. Spectral peaks similar to the surface peaks were found, suggesting that most waves propagate from the surface to the bottom in our simulations. Note that the energy content of the waves at the bottom is lower than that at the surface. This decrease in energy is expected since, under realistic stratification, the magnitude of the horizontal velocity eigenfunction for the first baroclinic mode is much greater at the surface than it is at the bottom for all waves (this is also true to a lesser extent for the second baroclinic mode). Dissipation of energy as the
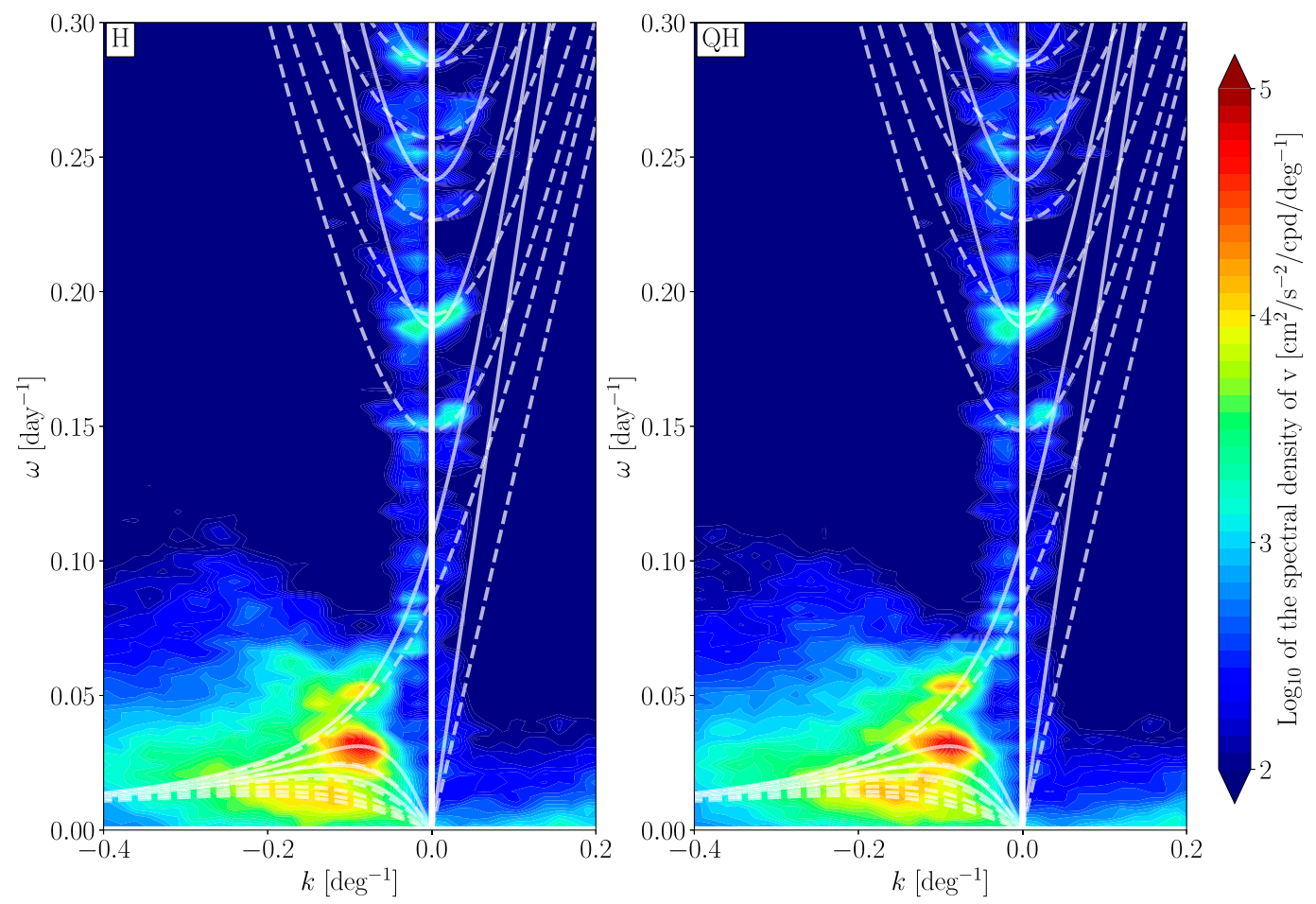

FIG. 4. Wavenumber-frequency power spectrum of the meridional velocity in the (left) $\mathrm{H}$ and (right) $\mathrm{QH}$ simulations averaged over the surface $\sigma$ layer on the whole numerical domain of the child simulation and over the five years of simulation. Solid and dashed white lines are the theoretical dispersion curves for baroclinic modes 1 and 2 , respectively, and are based on gravity wave speeds of 2.7 and $1.7 \mathrm{~m} \mathrm{~s}^{-1}$, respectively. 


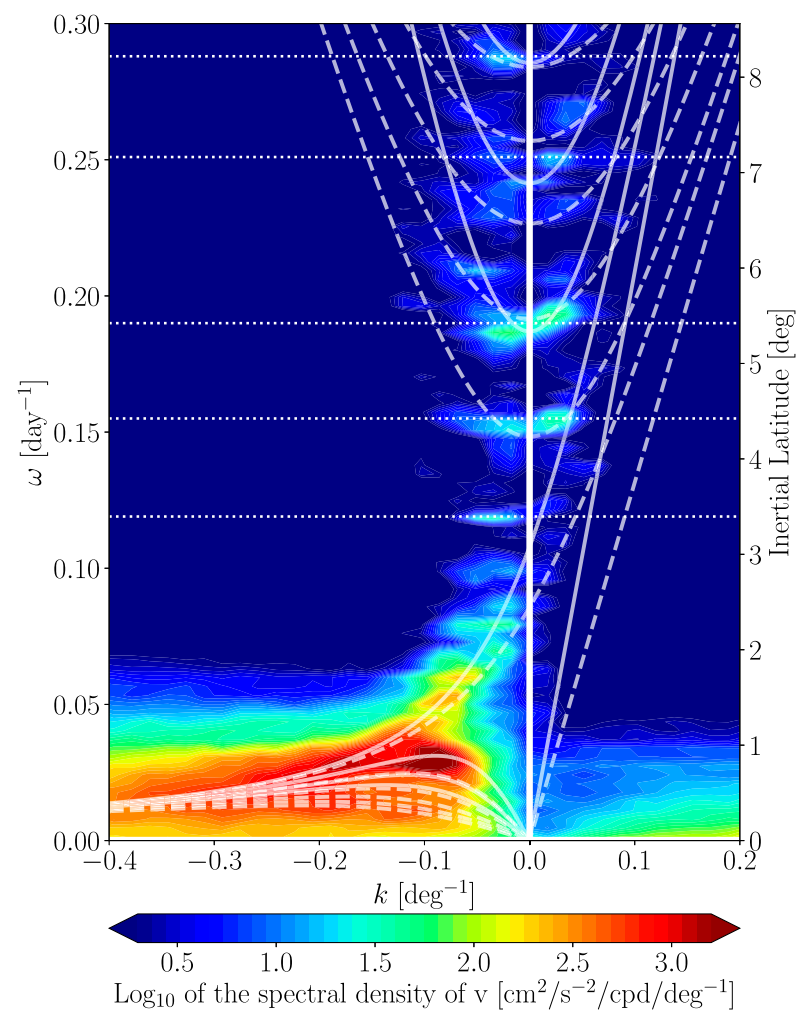

FIG. 5. As in Fig. 4, but for the meridional velocity averaged over the bottom $\sigma$ layer. The inertial latitude associated with each frequency is shown on the right $y$ axis. The horizontal dotted lines highlight the frequency and inertial latitudes of the waves that contribute the most to the enhanced shear in the $\mathrm{QH}$ run shown in Fig. 10.

waves are propagating down in the water column might also be at play. In addition, the relative magnitude of each peak is different, suggesting that some waves have either been dampened more than others as they propagate down, or that some waves have left or entered the domain of the simulation through the lateral boundaries at depth. For example, the 17-day Yanai wave is weaker than the 20-day Yanai wave in the abyss but stronger at the surface. Rossby and Yanai waves with relatively large zonal wavenumbers are present in the bottom spectrum yet absent near the surface. This enhancement of energy at shorter scales might be due to the presence of the bottom topography, which can lead to scattering and thus generate higher wavenumbers.

Overall, these results show that our simulations reproduce well the ETW field seen in the observations. In addition, these waves propagate down into the deep ocean, hence providing a realistic forcing mechanism for assessing the presence or lack thereof of critical reflection induced by NT effects, as predicted by DT19.

\section{b. Abyssal mixing}

We also contrasted the turbulent diffusivity in the $\mathrm{H}$ and $\mathrm{QH}$ simulations averaged over the bottom $1000 \mathrm{~m}$ of the water column and over the 5 years of simulation (Fig. 6).
In the $\mathrm{H}$ case, the diffusivity is enhanced between $\pm 2^{\circ}$ where the currents are the strongest and in areas of rough topography. The diffusivities are particularly large in the western part of the domain because of the presence of rough topography (see Fig. 1b). In the QH case, however, elevated diffusivities are also found outside of the $\pm 2^{\circ}$ equatorial band, with enhanced values extending from $8^{\circ} \mathrm{S}$ to $14^{\circ} \mathrm{N}$. Most of the high diffusivity values are found between $120^{\circ}$ and $155^{\circ} \mathrm{W}$ in the $\mathrm{QH}$ run. In the eastern part of the domain, the $\mathrm{QH}$ and $\mathrm{H}$ simulations look very similar.

Locations with higher diffusivities are associated with enhanced bottom shear in the simulations (Fig. 7). While most of the vertical shear is confined to $\pm 2^{\circ}$ or over rough topography in the $\mathrm{H}$ case, large values of the shear are seen over smooth topography and outside the equatorial band in the QH case. In some of these areas, the vertical shear in the $\mathrm{QH}$ simulation is more than 100 times greater than that in the $\mathrm{H}$ simulation. The locations of enhanced shear and subsequent mixing in the $\mathrm{QH}$ run match notably areas where $\Gamma \leq 1$ (shown within the contour line in Figs. 6 and 7), consistent with the theory described in DT19.

To highlight the vertical extent of the mixing (Fig. 8) and the corresponding shear (Fig. 9), we focus on a section along the $130^{\circ} \mathrm{W}$ longitude line. The diffusivities are enhanced in a bottom boundary layer in the QH case comparable to the surface boundary layer, and extend for up to $300 \mathrm{~m}$ off the bottom. This bottom boundary layer is completely absent in the $\mathrm{H}$ simulation. Enhanced diffusivities are also found higher in the water column in the $\pm 2^{\circ}$ equatorial band but this is the case for both the $\mathrm{H}$ and $\mathrm{QH}$ simulations. As highlighted in Fig. 9, the diffusivities near the bottom in the $\mathrm{QH}$ case are associated with higher vertical shear. In the $\mathrm{H}$ case, the shear is confined to the surface and in the $\pm 2^{\circ}$ equatorial band in the abyss. The ratio of the shear in the $\mathrm{QH}$ and $\mathrm{H}$ simulations (bottom panel) shows more clearly the vertical extent of the enhanced shear in the $\mathrm{QH}$ simulation. Overall, enhanced values in the $\mathrm{QH}$ case can reach up to $1000 \mathrm{~m}$ off the bottom.

To understand better the properties of the shear that is generated in the $\mathrm{QH}$ simulation, we have calculated the frequency spectrum of the vertical shear in the bottom $500 \mathrm{~m}$ of the water column as a function of latitude at $130^{\circ} \mathrm{W}$ (Fig. 10). In the $\mathrm{H}$ simulation, there is enhanced energy at only low frequencies and close to the equator, whereas in the $\mathrm{QH}$ simulation, enhanced energy is seen at all latitudes. In the latter case, the spectrum peaks around the inertial frequency, showing that the shear that is generated in the QH simulation is near inertial. Within the near-inertial band, some frequencies are more energetic than others and correspond to specific inertial latitudes. These frequencies correspond to the frequencies of particular ETWs that we found in the deep in our simulations (see Fig. 5), suggesting that the ETWs are responsible for the enhanced mixing in the QH run. A major peak, for example, occurs at a frequency of around 0.19 day $^{-1}$ and corresponds to an IGW with an inertial latitude of $5.5^{\circ}$. In addition, it can be seen that the enhanced energy in the $\mathrm{H}$ run is mostly superinertial whereas it extends a bit in the subinertial region in the $\mathrm{QH}$ case, consistent with the subinertial propagation of IGWs allowed when the NT effects are active. 

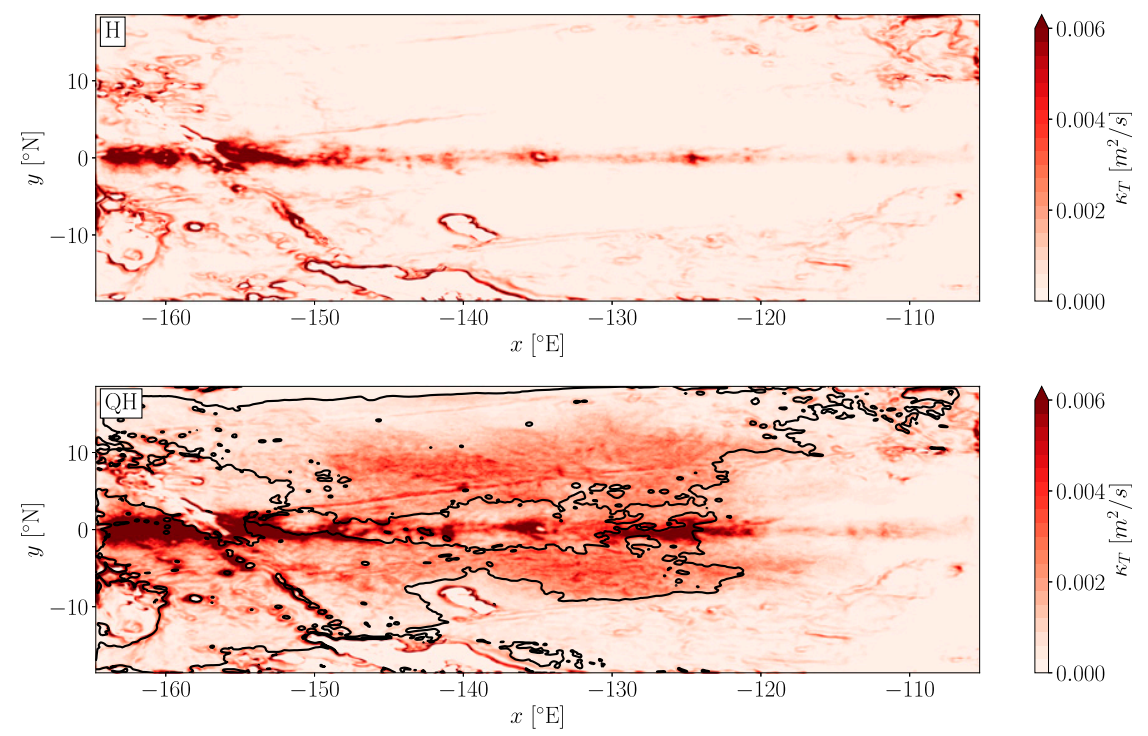

FIG. 6. The turbulent diffusivity averaged over the bottom $1000 \mathrm{~m}$ of the water column and over the 5 years of simulation in the (top) $\mathrm{H}$ and (bottom) $\mathrm{QH}$ runs. The black contour line in the bottom panel shows where $\Gamma=N / \tilde{f}=1$ at the bottom of the ocean over the bottom $\sigma$ layer; $\Gamma<1$ within the contour lines where enhanced turbulent diffusivity is found.

To better visualize the spatial and temporal variability of the shear, Hovmöller diagrams of the vertical shear of the meridional velocity as a function of latitude at $130^{\circ} \mathrm{W}$ are shown in Fig. 11. In the $\mathrm{QH}$ case, shear outside of the $\pm 2^{\circ}$ equatorial band forms stacked bands that oscillate near the local inertial frequency. The shear bands have a nonzero meridional wavenumber, with an equatorward phase velocity. However, the wave beams that are associated with these shear bands propagate poleward, away from their generation location. We highlight the vertical structure of the shear on the $5.5^{\circ} \mathrm{S}$ line (which corresponds to the inertial latitude of the 5-day wave) in Fig. 12. In particular, there is one high-shear event occurring between 15 December and 15 January with a downward phase propagation.

\section{Discussion}

In the previous section, we showed the diffusivities in the abyss were enhanced in a large part of the domain in the $\mathrm{QH}$ simulation compared to that in the $\mathrm{H}$ simulation. We further showed that the enhanced diffusivities were associated with inertial vertical shear that develops when $\tilde{f} \neq 0$. Our hypothesis is that the vertical shear is generated through the mechanism of critical reflection when ETWs reflect off the bottom, as predicted by DT19 theory.

It is possible to understand the theory better by looking at the governing equation for inviscid, zonally invariant, linear IGWs over an $f$ plane,

$$
\left(N^{2}+\tilde{f}^{2}\right) \psi_{y y}+2 \tilde{f} f \psi_{y z}+\left(f^{2}-\omega^{2}\right) \psi_{z z}=0
$$

where $\omega$ is the frequency of the wave, and $\psi$ is the streamfunction (i.e., $v=\partial \psi / \partial z$ and $w=-\partial \psi / \partial z$ ).
Equation (7) admits wavelike solutions where it is hyperbolic, i.e., where $B^{2}-A C>0$ with $A=N^{2}+\tilde{f}^{2}, B=\tilde{f} f$, and $C=f^{2}-\omega^{2}$. This constraint gives a lower bound for the frequency of the wave:

$$
\omega_{\min }=|f| \sqrt{1-\frac{\tilde{f}^{2}}{N^{2}+\tilde{f}^{2}}}=|f| \sqrt{\frac{\Gamma}{1+\Gamma}} .
$$

Note that, when $\tilde{f}=0$, we found the usual lower bound for IGWs under the traditional approximation: $\omega_{\min }=|f|$. With $\tilde{f} \neq 0$ and $\Gamma \leq 1$, values of $\omega$ that are significantly lower than $|f|$ are possible.

The characteristic curves of (7) represent rays along which wave energy propagates. The slope of these rays is equal to

$$
\mu^{ \pm}=\frac{B \pm \sqrt{B^{2}-A C}}{A} .
$$

To get the direction of energy propagation, we derive a dispersion relation using a plane wave solution $\psi(y, z) \sim e^{i(l y+m z)}$ in (7):

$$
\omega^{2}=f^{2}+2 \tilde{f} f \frac{l}{m}+\left(N^{2}+\tilde{f}^{2}\right) \frac{l^{2}}{m^{2}} .
$$

This is the dispersion relation for $2 \mathrm{D}$ inertia-gravity waves over an NT $f$ plane. Note that $\mu^{ \pm}=-l / m$.

Taking the gradient of (10) with respect to the wave vector yields the group velocity of the wave:

$$
\left(c_{g}^{y}, c_{g}^{z}\right)=\frac{l\left(N^{2}+\tilde{f}^{2}\right)+m f \tilde{f}}{m^{2} \omega}\left(1,-\frac{l}{m}\right),
$$

which gives the direction and speed of energy propagation, and constrains the characteristics along which the incoming and reflected waves propagate.

At the inertial latitude, $\mu_{\omega=f}^{ \pm}=0$ when $\tilde{f}=0$. However, $\mu_{\omega=f}^{+}=2 f / \tilde{f}(1+\Gamma)^{-1}$ in the NT case when $\tilde{f} \neq 0$. If $\Gamma$ is low 

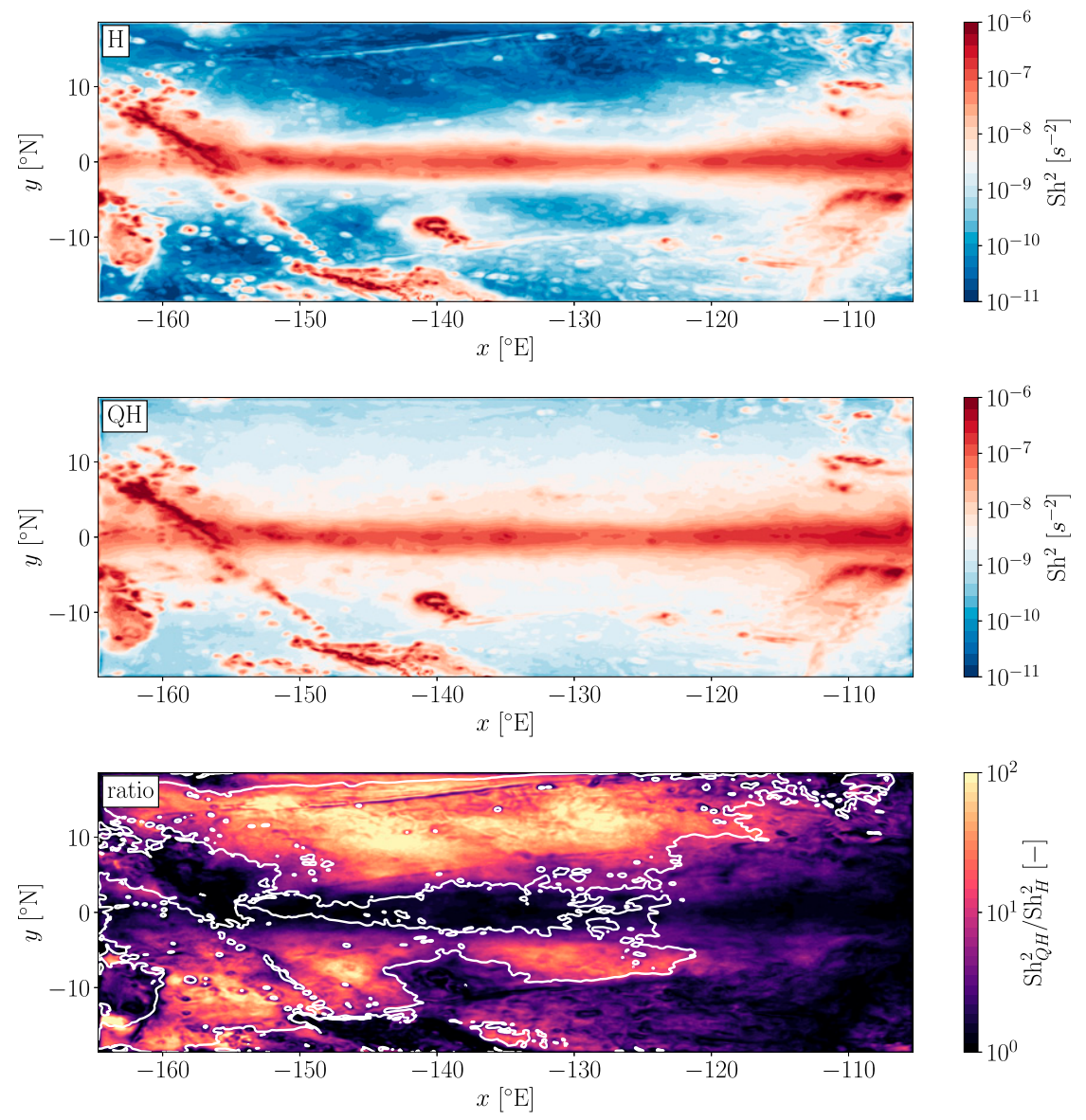

FIG. 7. As in Fig. 6, but for the vertical shear squared, $\mathrm{Sh}^{2}=(\partial u / \partial z)^{2}+(\partial v / \partial z)^{2}$. The ratio between the $\mathrm{QH}$ and $\mathrm{H}$ case is shown in the bottom panel. The white contour line in the bottom panel shows where $\Gamma=N / \tilde{f}=1$ at the bottom of the ocean over the bottom $\sigma$ layer; $\Gamma<1$ within the contour lines where enhanced vertical shear is found in the QH case.

enough, $\mu_{\omega=f}^{+}$is significant, allowing for vertical propagation of energy at the inertial latitude along the steep characteristic. Since $\mu_{\omega=f}^{-}=0$, the second characteristic is flat and therefore ray tubes becomes compressed to an infinitesimally small layer when reflecting off a flat bottom, resulting in critical reflection. This mechanism is illustrated in Fig. 13. It requires three key elements to occur: a downward flux of ETW energy along the steep characteristic, $\Gamma \leq 1$ such that $\mu_{\omega=f}^{+}$is significant, and a bottom slope low enough such that ray tubes are compressed enough to amplify the reflected wave.

In the next subsections, we discuss whether the conditions are met for critical reflection to occur. Then, we look at the properties of the shear that develops in the $\mathrm{QH}$ simulation to examine whether our hypothesis is consistent with the mechanism of critical reflection described above. Finally, we discuss the implications of the enhanced diffusivities for the abyssal stratification and diapycnal upwelling.

\section{a. Vertical propagation of equatorial waves}

To investigate whether ETWs can flux energy on the steep characteristics near the bottom where enhanced vertical shear is found, we selected six spectral peaks from Figs. 4 and 5 to track their vertical propagation of energy: the 33-day Rossby wave, the 23-day Yanai wave, the 17-day Yanai wave, the 6-day IGW, the 5-day IGW, and the 3-day IGW.

The location where these waves are generated at the surface can be identified by calculating the variance of the meridional velocity filtered around the frequency for each of the six waves (Fig. 14). Depending on the type of wave, maxima in the variance are found in different locations. The 3-day IGW is mostly generated near $125^{\circ}-140^{\circ} \mathrm{W}$ to the north of the equator, while the 5 -day IGW has a peak around $130^{\circ}-145^{\circ} \mathrm{W}$ on the south side of the equator. The 6-day IGW is more spread out, with a stronger signal on the southern side of the equator between $110^{\circ}$ and $150^{\circ} \mathrm{W}$. Both Yanai waves have peaks centered at the equator in the eastern side of our domain, between $110^{\circ}$ and $130^{\circ} \mathrm{W}$. The 33 -day Rossby wave is mostly generated in the Northern Hemisphere, between $120^{\circ}$ and $140^{\circ} \mathrm{W}$.

In Figs. 4 and 5, we have shown that the six waves considered here lie on the theoretical dispersion curves for baroclinic modes 1 and 2 . The dispersion relation for such waves was derived by Cox (1980): 

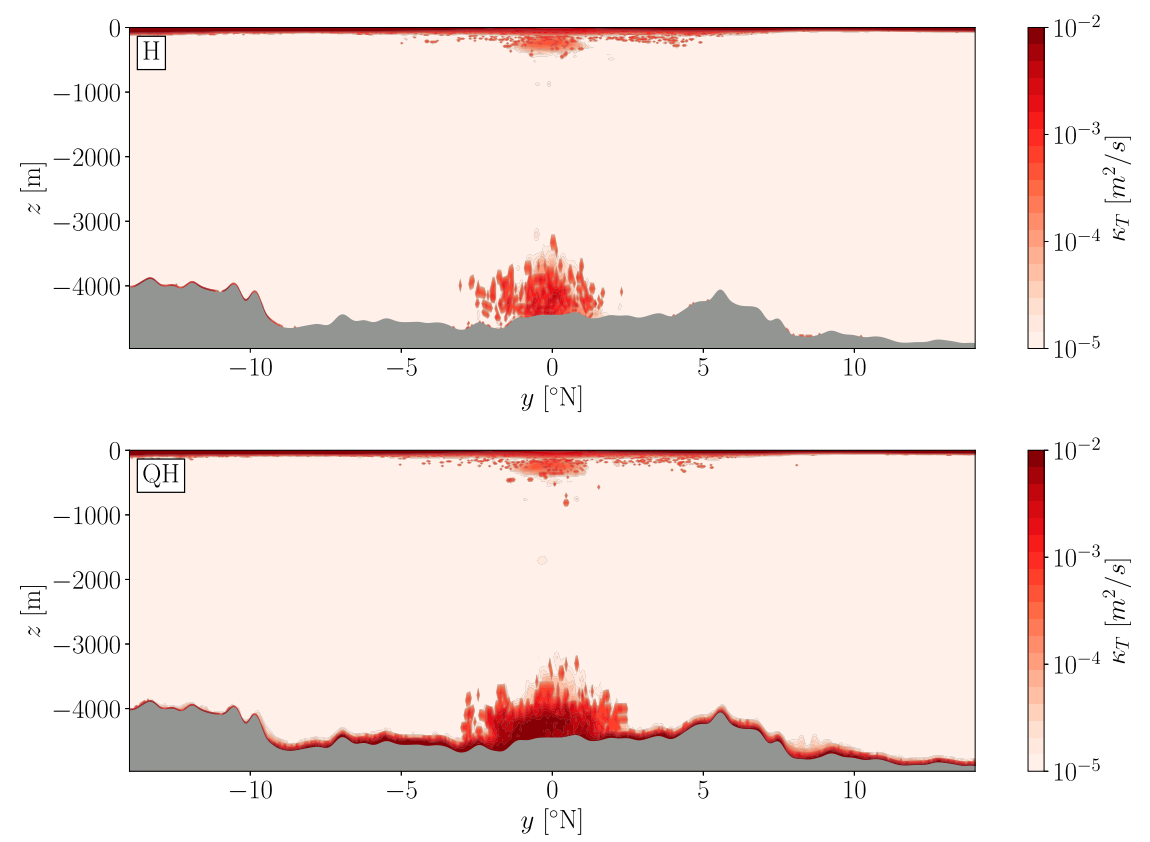

FIG. 8. Turbulent diffusivity from the (top) $\mathrm{H}$ and (bottom) QH simulations at $130^{\circ} \mathrm{W}$ averaged over the five years of simulation.

$$
c=\frac{-\beta(2 n+1) \pm \sqrt{\beta^{2}(2 n+1)^{2}+4\left(k^{2}+\beta k \omega^{-1}\right) \omega^{2}}}{2\left(k^{2}+\beta k \omega^{-1}\right)}
$$

where $c$ denotes the phase speed for the $n$th baroclinic mode Kelvin wave; $k$ and $\omega$ are the zonal wavenumber and frequency of the wave, respectively; and $\beta=2 \Omega / R_{E}$ is the meridional gradient of the vertical component of the Coriolis parameter at the equator ( $\Omega$ is Earth's angular velocity and $R_{E}$ is Earth's radius). The effects of variable stratification are manifest in the local wavenumber,

$$
m(z)=N(z) / c
$$

From Eqs. (12) and (13), it is possible to derive the expressions for the zonal group velocity $c_{g}^{x}$ and the vertical group velocity $c_{g}^{z}$ :

$$
\begin{aligned}
c_{g}^{x} & =\frac{c^{2}\left(2 k+\beta \omega^{-1}\right)}{2 \omega+\beta k c^{2} \omega^{-2}}, \\
c_{g}^{z} & =-\frac{\omega^{2}+\beta k c^{2} \omega^{-1}+k^{2} c^{2}}{N\left(2 \omega c^{-1}+\beta k c \omega^{-2}\right)} .
\end{aligned}
$$

We used Eqs. (14) and (15) to quantify the pathways of propagation for the six waves we have considered. For each wave, we randomly generated a range of frequencies and zonal wavenumbers that are appropriate for the wave of interest. We used the map of the variance of the meridional velocity at the surface for each wave (Fig. 14) to construct a probability distribution for the starting point of the rays, with higher probabilities in regions where the variance is larger. We used the stratification profile measured by Holmes et al. (2016) in the eastern equatorial Pacific and shown in Fig. 2 to trace the rays.
For each wave, we launched 20 rays (Fig. 15). Our results show that the Rossby and IGW rays are nearly vertical while the Yanai waves propagate away from their generation longitude to the east. In our calculation, most of the IGW and Rossby wave rays reach the bottom between $125^{\circ}$ and $145^{\circ} \mathrm{W}$, which is the area where we see the largest diffusivities in the $\mathrm{QH}$ run. The IGWs are in particular confined to this longitude band, which potentially explains the enhanced diffusivities that develop at higher latitudes since their inertial latitudes lie further away from the equator. The Yanai waves propagate eastward, and most of them reach the seafloor out of our domain. Note that depending on the type of wave, the travel time from the surface to the bottom varies greatly: while the IGW reach the seafloor within a few days, it can take several months for the Yanai and Rossby waves to hit the bottom.

\section{b. Bottom slope and the slope of the steep characteristic}

The two other key ingredients needed for critical reflection are linked to the slope of the seafloor and the slope of the steep characteristic at the inertial latitude of the wave, $\mu_{\omega=f}^{+}$.

If the steep characteristic has a slope that is too low, the compression of ray tubes will be limited, weakening amplification by the critical reflection mechanism. Similarly, if the slope of the seafloor is too high, the compression of ray tubes upon reflection is reduced and the critical reflection mechanism is not as effective. Therefore, a key metric for the effectiveness of the critical reflection mechanism is the ratio of $\mu_{\omega=f}^{+}$ to the slope of the seafloor, which we plot in Fig. 16. Over most of the domain, the ratio is large, suggesting that the mechanism of critical reflection is efficient there. The ratio is small over rough topography as expected. However, it is also small at the equator. To understand why, consider the limit when $\Gamma \ll 1$, 

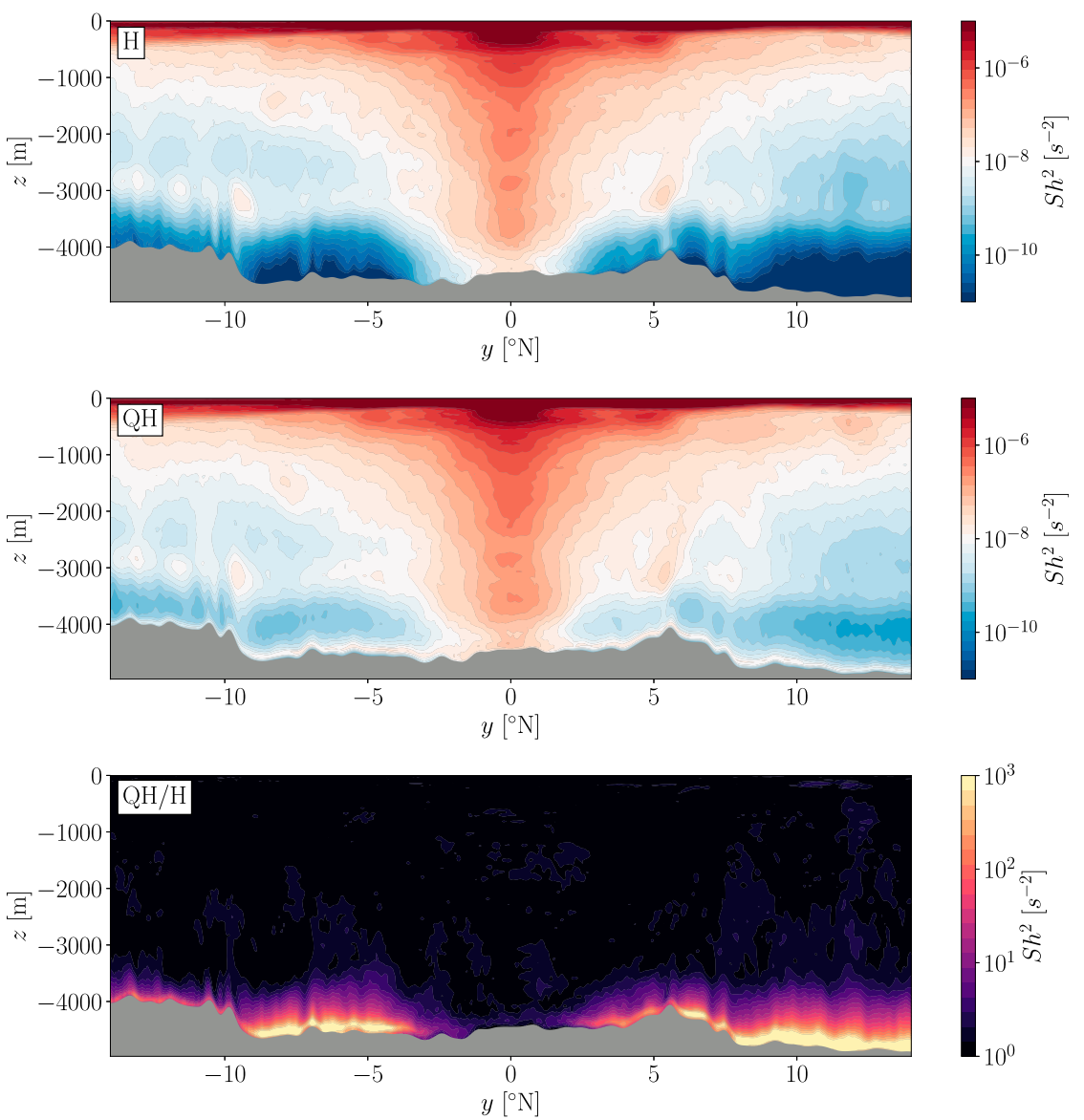

FIG. 9. As in Fig. 8, but for the vertical shear squared, $\mathrm{Sh}^{2}=(\partial u / \partial z)^{2}+(\partial v / \partial z)^{2}$. The ratio between the $\mathrm{QH}$ and $\mathrm{H}$ case is shown in the bottom panel.

which is the case in most parts of the domain at the bottom. In this limit, the slope of the steep characteristics becomes $\mu_{\omega=f}^{+}=2 f / \tilde{f}$. Close to the equator, this expression is approximately to $\mu_{\omega=f}^{+} \approx 2 \phi$, where $\phi$ is the latitude. Therefore, close to the equator, the mechanism of critical reflection is expected to be less effective than away from the equator. This was observed in the idealized simulations of DT19 where it was seen that the longer the period of the ETW and hence the lower the inertial latitude, the weaker the critical reflection. We appear to see this effect in our realistic simulations as well. For example, the ratio of the vertical shear squared in the $\mathrm{QH}$ to the $\mathrm{H}$ simulation increases with latitude (Fig. 7). In addition, we see that the higher frequency ETW result in stronger shears. For example, the most visible beams of shear seen in Fig. 11 originate from $5.5^{\circ}$ and $8^{\circ}$, which correspond to the inertial latitudes of the 3-and 5-day IGW that we see at the surface and at the bottom in our simulations (Figs. 4 and 5).

We should note, however, that beyond a certain latitude (around $10^{\circ}$ in the Northern Hemisphere and $8^{\circ}$ in the Southern Hemisphere), the ratio of the vertical shear decreases again. It also exhibits an asymmetry in the east-west direction, suggesting that factors other than latitude come into play. In particular, $\mu_{\omega=f}^{+}$depends on $\Gamma$ which is a function of both the stratification and $\tilde{f}$. While $\tilde{f}$ decreases with latitudes, the abyssal stratification can vary laterally. These two factors can lead to areas where $\Gamma>1$ at the bottom. We highlight these regions in Figs. 6 and 7, where they are delineated with the $\Gamma=$ 1 contour. As predicted by the theory, the enhanced vertical shear and mixing occur over areas where $\Gamma<1$. In the Southern Hemisphere, this area extends down to $10^{\circ} \mathrm{S}$. In the Northern Hemisphere however, $\Gamma$ remains below one past $10^{\circ} \mathrm{N}$. In the simulation featured in this paper, the domain is not big enough to determine the meridional limit where $\Gamma \leq 1$. However, we have run a lower-resolution simulation that extends to $\pm 20^{\circ} \mathrm{N}$ and that suggests that values of $\Gamma<1$ are found up to $18^{\circ} \mathrm{N}$ in the eastern equatorial Pacific.

\section{c. Properties of the vertical shear}

The analyses described above suggest that the key elements needed for critical reflection are met in the $\mathrm{QH}$ run and coincide with the areas where shear is enhanced. To rigorously associate the enhanced shear with DT19 theory, however, we still need to demonstrate that the properties of the shear that develops in our simulation are consistent with the ones predicted by DT19.

First, as shown in Fig. 10, the vertical shears in the QH simulation with the most energy have near-inertial frequencies. This is consistent with DT19 theory since critical reflection preferentially amplifies shear at the inertial latitude. As evident in 

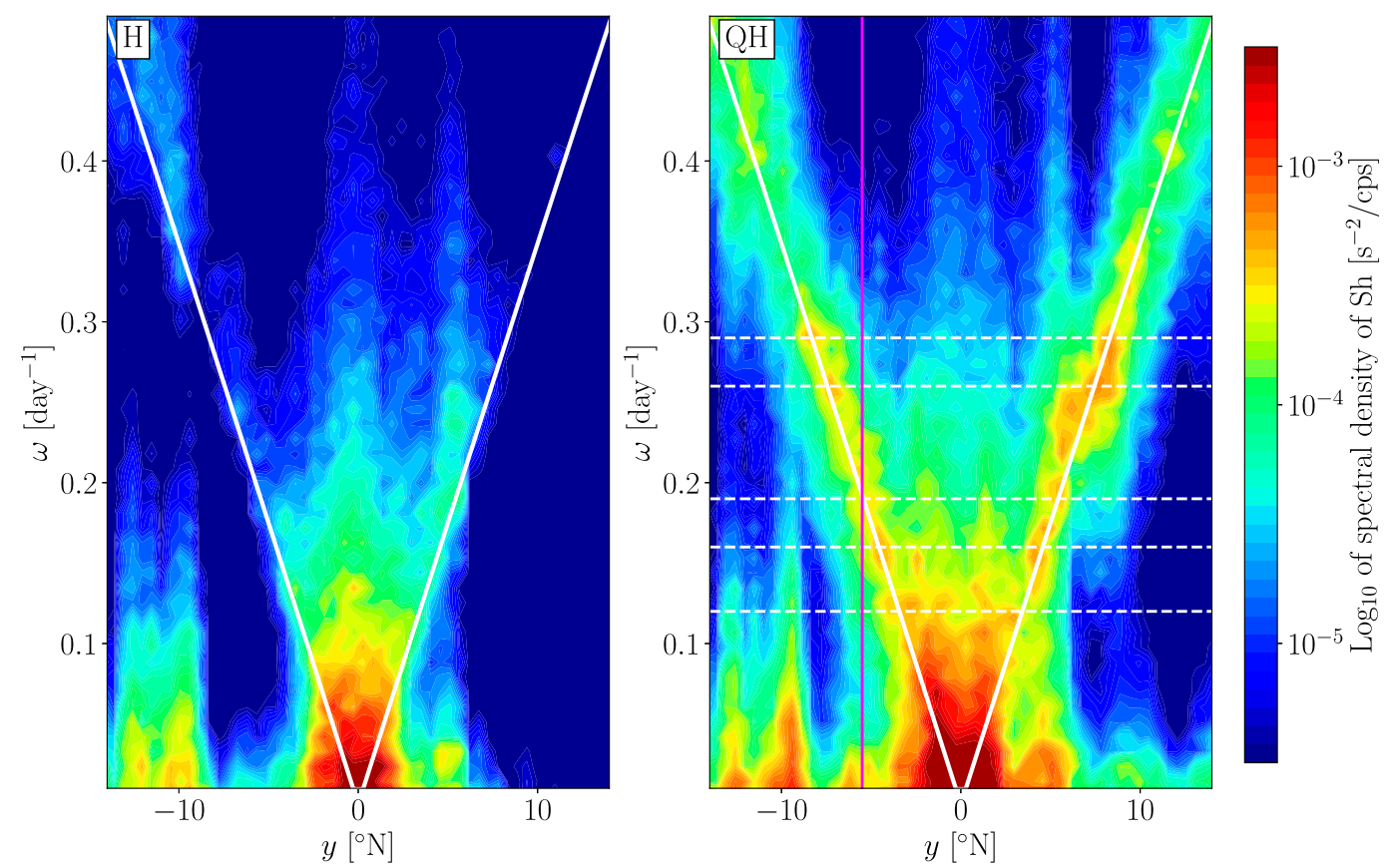

FIG. 10. Spectrum of the vertical shear in the bottom $500 \mathrm{~m}$ of the water column from the (left) $\mathrm{H}$ and (right) QH simulations at $130^{\circ} \mathrm{W}$ as a function of latitude. The spectrum has been calculated over the 5 years of simulation. The plain white lines correspond to the inertial frequency. The horizontal dotted lines highlight the frequency of the peaks in the spectrum of the shear in the $\mathrm{QH}$ run. The vertical magenta line indicates the latitude $5.5^{\circ} \mathrm{S}$ along which significant beams are found in Fig. 11.

Fig. 11, these inertial waves have a nonzero horizontal wavenumber. Although this is not permitted for traditional IGWs, it is allowed when $\tilde{f} \neq 0$. From (10), $\omega=f$ when $l=0$, or when $l / m=-2 f \tilde{f} /\left(N^{2}+\tilde{f}^{2}\right)$, which yields a meridional wavelength, $\lambda_{y}=\lambda_{z} N^{2}+\tilde{f}^{2} / 2|f| \tilde{f}$, where $\lambda_{z}$ is the vertical wavelength of the wave. From Fig. 12, we observe that for the first beam $\lambda_{z}=500 \mathrm{~m}$. Using the value of the stratification, $|f|$ and $\tilde{f}$ at the latitude of the beam, the factor $\left(N^{2}+\tilde{f}^{2}\right) /(2|f| \tilde{f})$ is equal to $\sim 4 \times 10^{2}$, which would yield a meridional wavelength of $\lambda_{y} \sim 20 \mathrm{~km}\left(\sim 0.2^{\circ}\right)$. This value is similar to the lateral wavelength of the beam seen in Fig. 11.

Apart from oscillating at frequencies near $f$, the shear has a very particular phase propagation. As illustrated in Fig. 11, near $5^{\circ} \mathrm{S}$ for example, phase propagates equatorward while energy propagates poleward (i.e., the direction of beam propagation). This apparent opposition of the phase and group velocity in the meridional direction is consistent with the theory for IGWs when NT effects are taken into account.

A schematic of the wave characteristics with their corresponding group and phase velocity vectors for $\tilde{f} \neq 0$ in the superinertial $(\omega>f)$, inertial $(\omega=f)$, and subinertial limits $(\omega<f)$ illustrates this point (Fig. 17). Note that this schematic is for IGWs in the Northern Hemisphere. We first focus on the subinertial limit since the beams propagate poleward of their inertial latitude and hence become subinertial, which is allowed when NT effects are taken into account. The behavior of these waves is very different than IGWs under the traditional approximation, that is, the phase and group velocity take the same sign in the vertical and the opposite sign in the meridional on the shallow characteristic (their behavior on the steep characteristic is similar to that of traditional IGWs). As described in DT19 (see their Fig. 5), after critical reflection, IGWs propagate away from the inertial latitude poleward and upward along the shallow characteristic (blue line). Once the wave reaches the separatrix where the wave equation transitions from hyperbolic to elliptic, it reflects back down on the steep characteristic until it reaches the seafloor and reflects back up on the shallow characteristic, and so on. Therefore, while the direction of energy propagation changes every time the wave switches from one characteristic to the other, the meridional phase propagation always points toward the equator. In the superinertial regime, the reflected wave propagates upward and equatorward along the shallow characteristic. In this case, we have also an equatorward phase propagation, potentially explaining why all the beams that we see in Fig. 11 have an equatorward phase propagation.

Note also that the vertical phase propagation of the first beam event in Fig. 12 is downward. We can see (Fig. 11) that the latitude chosen to plot the vertical section in Fig. 12 is slightly equatorward of the first beam event. Therefore, we are at the border of the superinertial regime for this beam event. In the superinertial regime, where the reflected wave propagates upward along the shallow characteristic, the phase propagation is downward (Fig. 17), consistent with what we see in the simulation.

\section{d. Implication for abyssal stratification and upwelling}

The enhanced diffusivities in the abyss associated with NT effects have two main consequences: weakening the stratification 

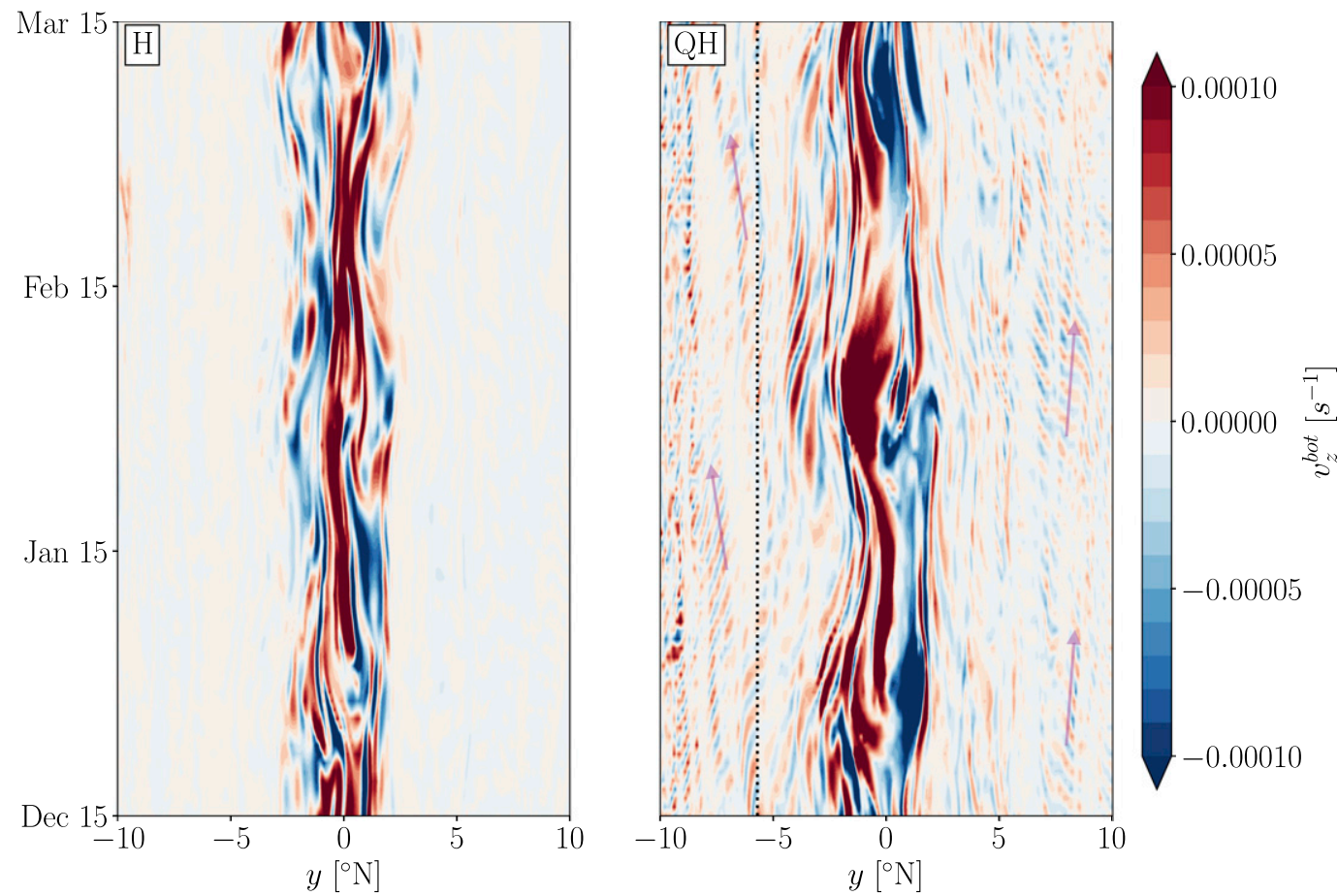

FIG. 11. Hovmöller diagram of time vs latitude of the vertical shear of the meridional velocity at $130^{\circ} \mathrm{W}$ for the three last months of the (left) $\mathrm{H}$ and (right) $\mathrm{QH}$ simulations at the bottom of the water column. The black dotted line shows the latitude $5.5^{\circ} \mathrm{S}$ along which the vertical profiles in Fig. 12 were made. The pink arrows indicate the energy propagation of the beams.

and driving diapycnal motions. To quantify the differences in stratification cause by NT effects, we calculated histograms of the buoyancy frequency in the bottom $1000 \mathrm{~m}$ of the water column and contrasted the distributions in the $\mathrm{H}$ and $\mathrm{QH}$ runs
(Fig. 18). The histograms have been calculated across the entire domain. We can see that while the mean and median values of the distributions are similar in the $\mathrm{H}$ and $\mathrm{QH}$ runs, the 10th percentile is much lower in the $\mathrm{QH}$ case (about two orders of

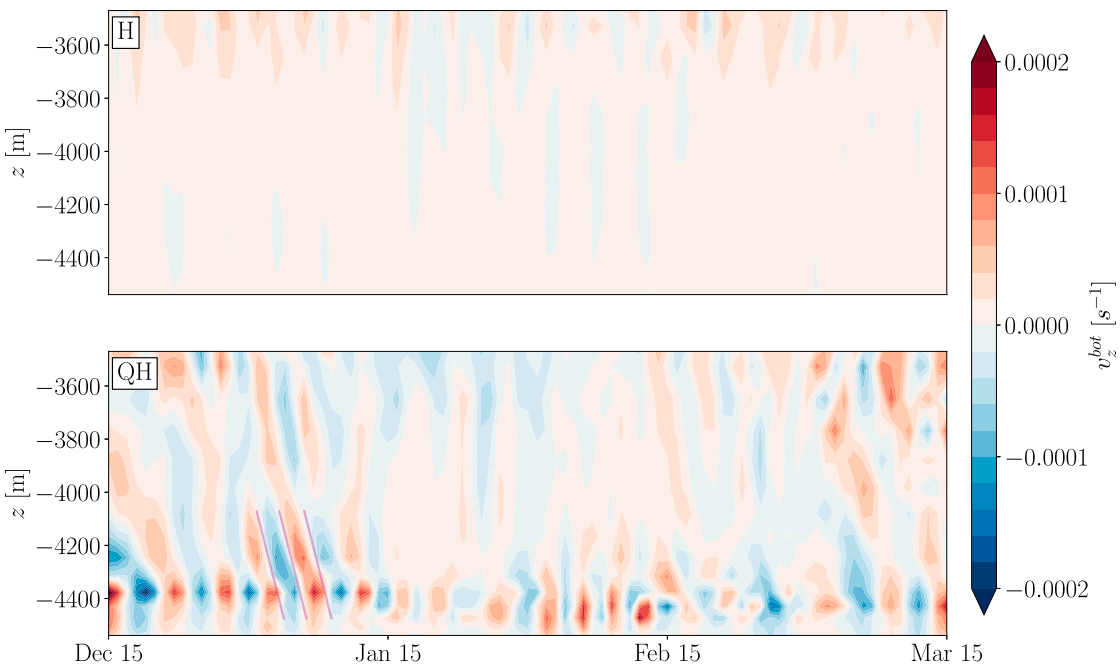

FIG. 12. Hovmöller diagram of depth vs time of the vertical shear of the meridional velocity at $130^{\circ} \mathrm{W}$ and $5.5^{\circ} \mathrm{S}$ (corresponding to the black dotted line in the right panel of Fig. 11) for the last 3 months of the (top) $\mathrm{H}$ and (bottom) $\mathrm{QH}$ simulations. The pink lines emphasize the vertical tilt in the phase of the beams. 


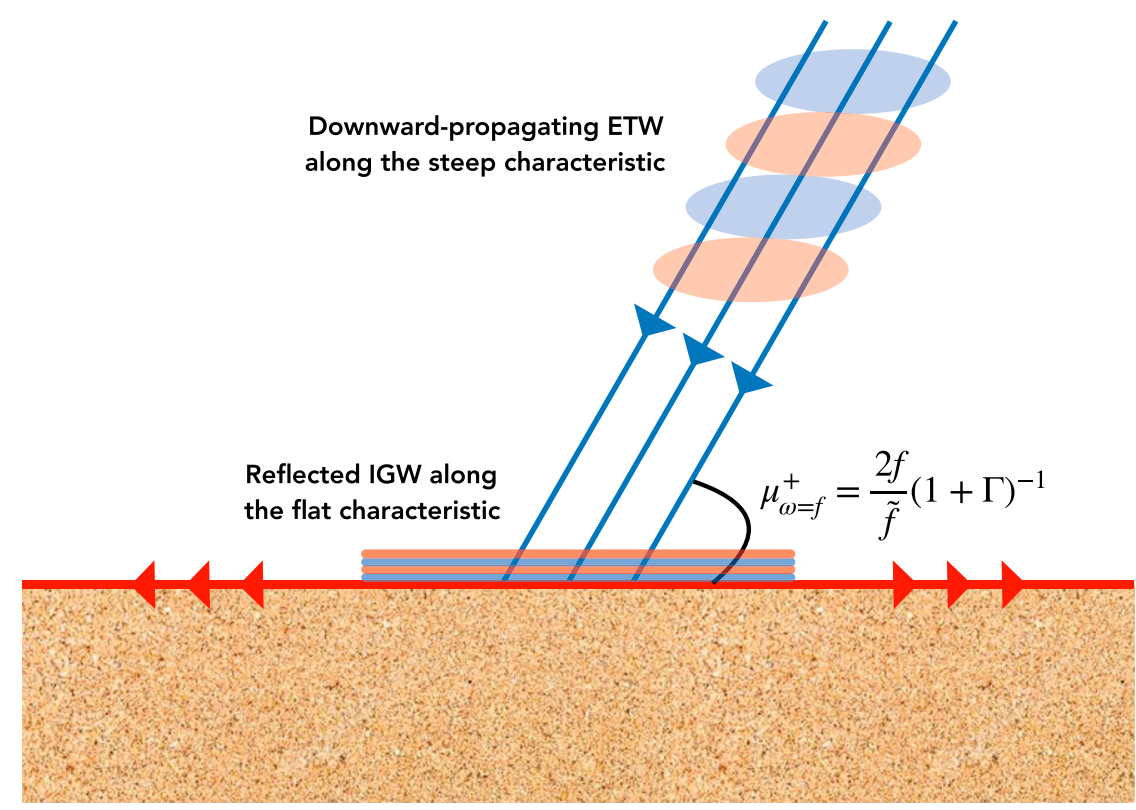

FIG. 13. Schematic of the critical reflection mechanism. A downward-propagating ETW transmits energy on the steep characteristic, which has a slope $\mu_{\omega=f}^{+}$. At a flat boundary, ray tubes are compressed to an infinitesimally small layer resulting in an amplification of the reflected IGW at the inertial latitude of the wave. The blue and red contours are depicting the phase of the wave.

magnitude lower in some regions), suggesting that NT effects act to skew the distribution toward low values in areas where there are enhanced diffusivities.

To assess the impact that the enhanced diffusivities that develop in the abyss may have on the diapycnal transport of water masses, we calculate the diabatic vertical velocity, defined as

$$
w_{\mathrm{dia}}=\left(\frac{\partial \kappa}{\partial z} N^{2}+\kappa \frac{\partial N^{2}}{\partial z}\right) / N^{2}
$$
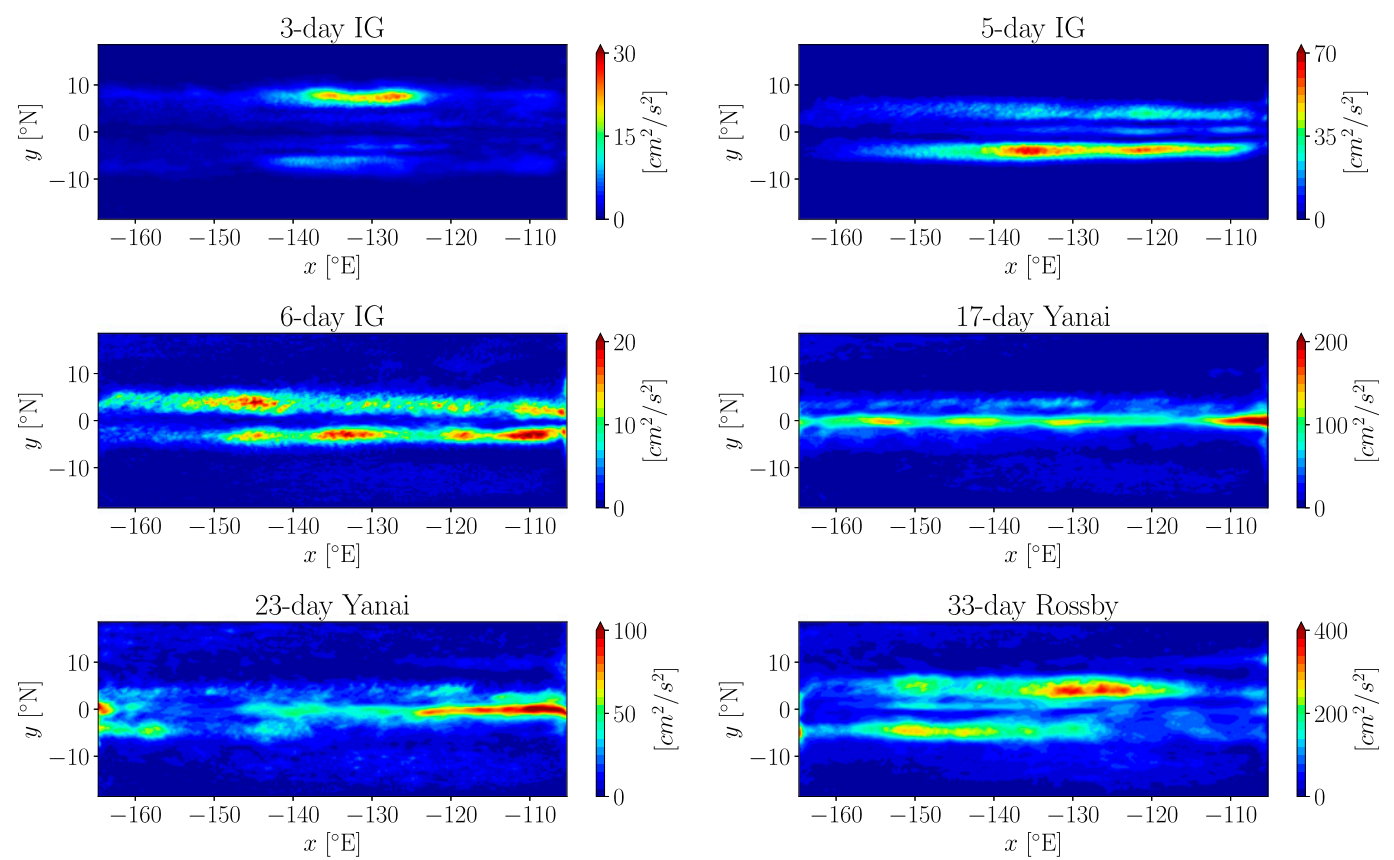

FIG. 14. Variance of the surface meridional velocity bandpass filtered around the frequency of six ETWs seen in Figs. 4 and 5: the 33-day Rossby wave, the 23-day Yanai wave, the 17-day Yanai wave, the 6-day IGW, the 5-day IGW, and the 3-day IGW. 


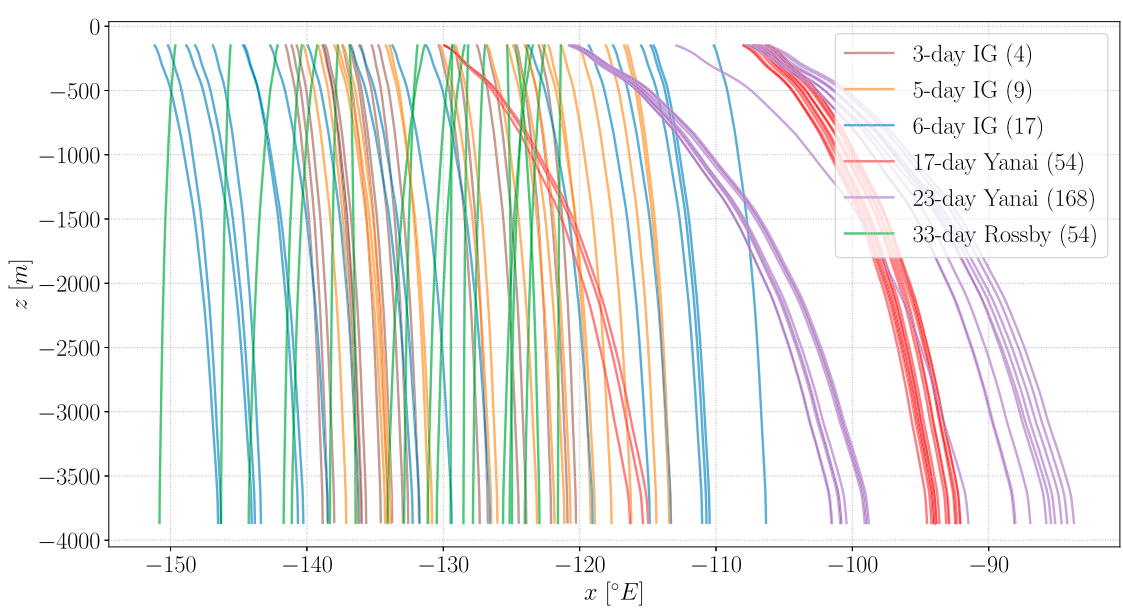

FIG. 15. Ray paths of the six ETW types in Fig. 14. For each wave, 20 rays are fired from the surface. The frequency and zonal wavenumber of the waves are chosen randomly within a range corresponding to each wave type. Similarly, the starting longitude of the wave is chosen randomly within an area corresponding to enhanced surface velocity variance for each wave (shown in Fig. 14). The number in parentheses next to the name of the wave corresponds to the average time in days it takes for the wave to reach the bottom from the surface.

Histograms of the diapycnal vertical velocity in the bottom $1000 \mathrm{~m}$ of the water column for the $\mathrm{H}$ and $\mathrm{QH}$ runs are contrasted in Fig. 19. It can be seen that while most values are near zero in the $\mathrm{H}$ case (except near the equator where there is a nearly symmetric distribution of positive and negative diapycnal velocities), the distribution is skewed toward positive values in the $\mathrm{QH}$ case in areas where there is enhanced mixing. This effect is more clear when looking at the mean of the distribution, which is very close to zero in the $\mathrm{H}$ run except right at the equator where it is slightly positive, while it is skewed to positive values in the $\mathrm{QH}$ run in areas where enhanced diffusivities have been found. While the diffusivities are bottom-intensified, they induce diapycnal upwelling not downwelling because the stratification increases with height off the bottom (e.g., Fig. 2) and the second term in (16) dominates over the first.
We have integrated the diapycnal vertical velocity across the entire numerical domain of the child grid to calculate a vertical volume transport quantity $\Psi_{\mathrm{dia}}$ as

$$
\Psi_{\mathrm{dia}}=\iint w_{\mathrm{dia}} d x d y
$$

which is expressed in units of Sverdrups (Sv). We found $\Psi_{\text {dia }}^{H}=2.5 \mathrm{~Sv}$ and $\Psi_{\text {dia }}^{\mathrm{QH}}=23 \mathrm{~Sv}$, suggesting that the process could contribute substantially to the AMOC.

\section{e. Impact of the bottom drag}

To investigate whether our results hold in the presence of bottom drag, we have run the same $\mathrm{H}$ and $\mathrm{QH}$ simulations as before except that we allowed for bottom stress which was parameterized using a quadratic drag law. Bottom drag

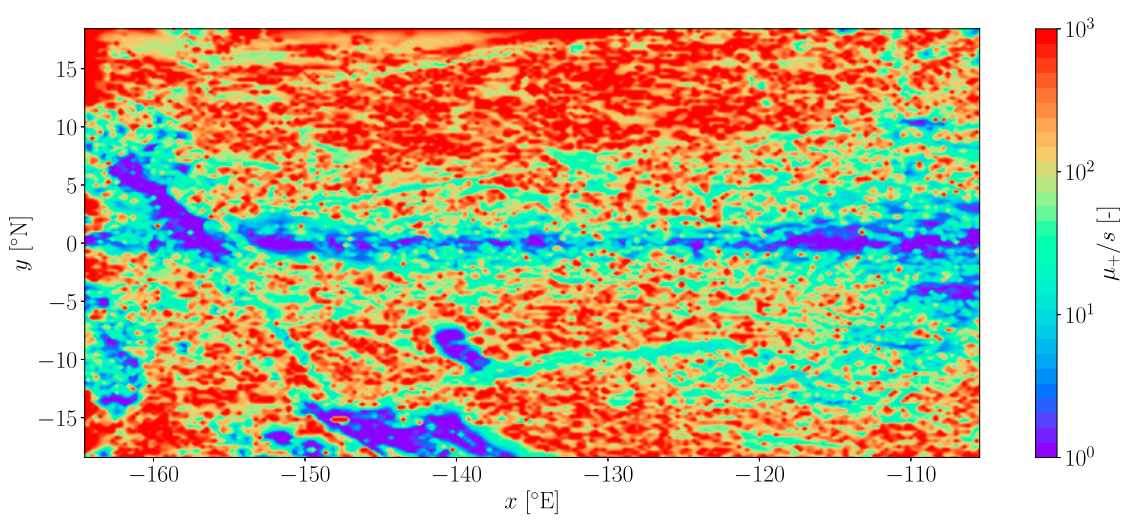

FIG. 16. Ratio of the slope of the steep characteristic at the inertial latitude, $\mu_{\omega=f}^{+}$(as defined in Fig. 13), to the slope of the seafloor in the meridional direction, $s=\partial h / \partial y$, where $h$ is the depth of the water column. When this ratio is large, the critical reflection mechanism due to NT effects is more efficient. 

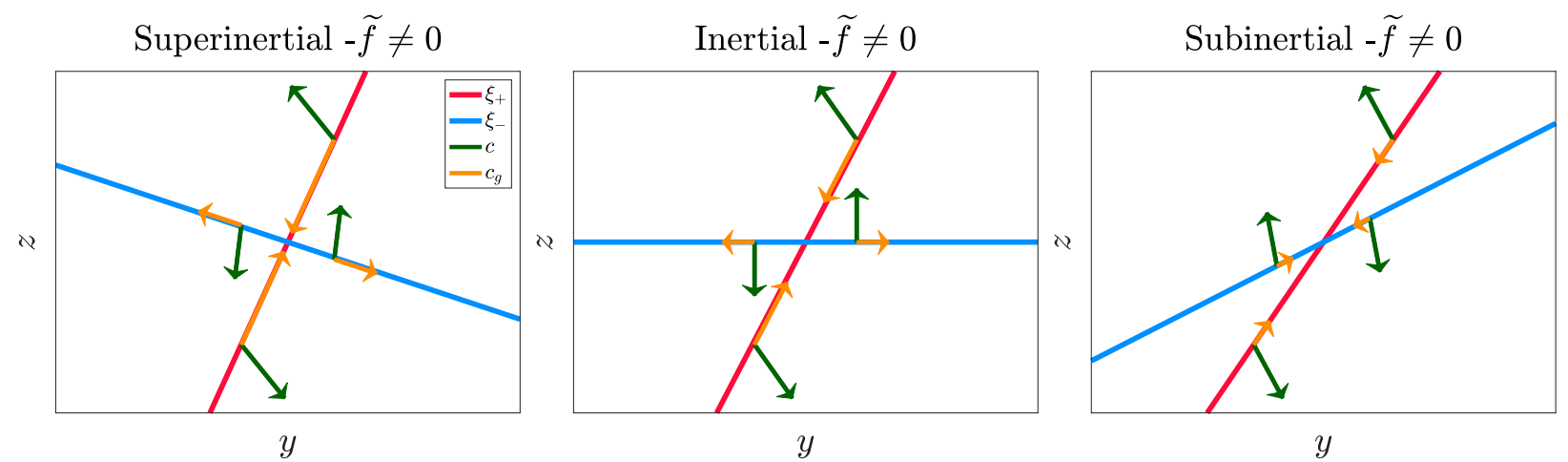

FIG. 17. Characteristic curves $\xi_{ \pm}=\mu^{ \pm} y-z$ (in blue and red), phase velocity vectors (in green) and group velocity vectors (in orange) for an inertia-gravity wave propagating over an $f$ plane with constant stratification in the NT case in the (left) superinertial $(\omega>f)$, (center) inertial $(\omega=f)$, and (right) subinertial $(\omega<f)$ regimes (where $\omega$ the frequency of the wave).

enhances the vertical shear, making it more difficult to distinguish the shear generated by critical reflection. However, there is a distinguishable difference in diffusivities between the $\mathrm{QH}$ and $\mathrm{H}$ simulations even in the presence of drag. We illustrate this using the turbulent diffusivity averaged over the bottom $1000 \mathrm{~m}$ of the water column, over all longitudes, and over the 5 years of simulation (Fig. 20). It can be seen that the enhancement in diffusivities in the $\mathrm{QH}$ simulations relative to the $\mathrm{H}$ simulations is similar with or without drag, showing that NT effects are evident even when bottom drag is active.

\section{Conclusions}

By contrasting realistic numerical simulations of the eastern equatorial Pacific Ocean run with the hydrostatic and quasihydrostatic approximations and theory, we have shown that NT effects modify the dynamics of ETWs in the deep. In the weakly stratified abyss, the reflection of ETWs off the bottom are modified by the NT effects, and the waves undergo critical reflection at their inertial latitude. As a consequence, strong inertial vertical shear is generated and drives mixing that is enhanced primarily between $120^{\circ}-150^{\circ} \mathrm{W}$ and $8^{\circ} \mathrm{S}-14^{\circ} \mathrm{N}$.

There are still some questions that remain to be answered. In particular, the presence of enhanced shear and subsequent mixing at relatively high latitudes (between $3^{\circ}-8^{\circ} \mathrm{S}$ and $3^{\circ}-14^{\circ} \mathrm{N}$ ) suggests that IGW with higher frequencies are found near the bottom in these areas. Whether these waves are generated by surface forcing or correspond to higher harmonics of the dominant ETWs formed by nonlinear wave-wave interactions as was seen by DT19 remains to be investigated.

Overall, these results show that NT effects could contribute significantly to the diapycnal upwelling of the abyssal equatorial circulation inferred by inverse models. Our study is however limited by the Richardson number-dependent mixing scheme that we used, which parameterizes the strength of the turbulent diffusivity. Further work will focus on using turbulence-resolving simulations to study mixing driven by critical reflection and to parameterize its effects. In addition, because the shape of the stratification profile in
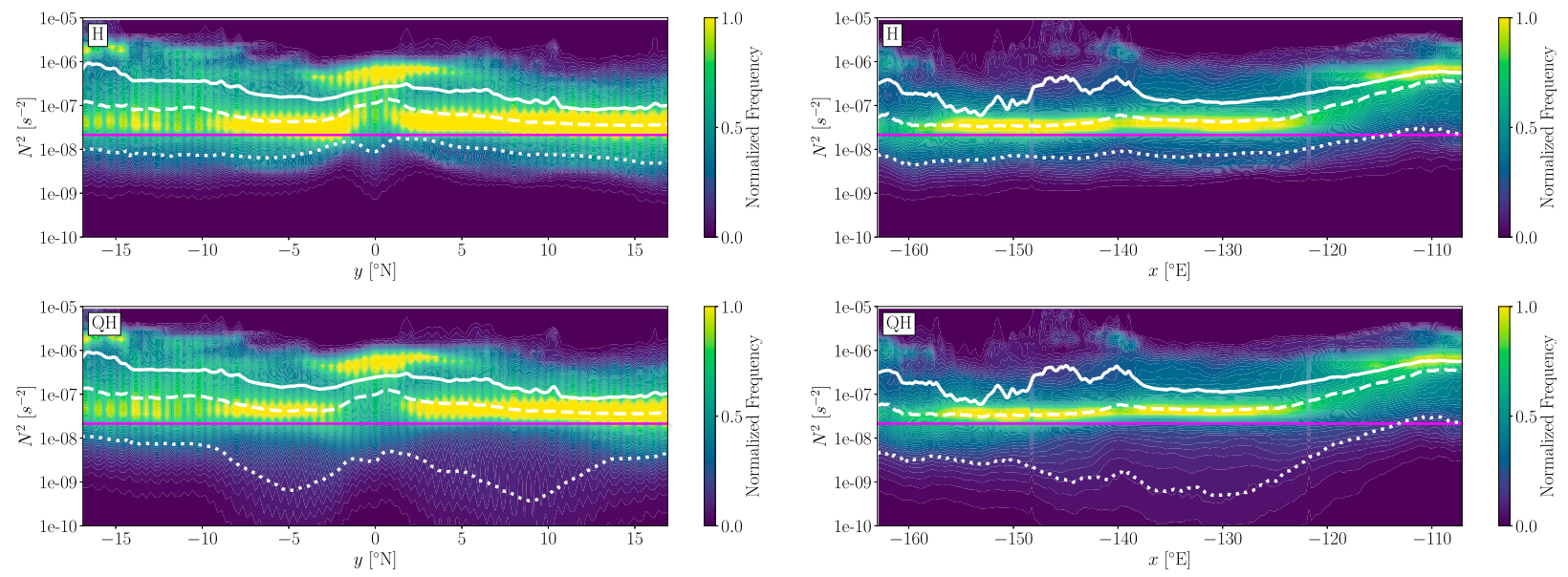

FIG. 18. Histogram of the buoyancy frequency in the bottom $1000 \mathrm{~m}$ of the water column with the mean (plain white line), median (dashed white line), and 10th percentile (dotted white line) of the distribution plotted as a function of (left) latitude and (right) longitude for the (top) $\mathrm{H}$ and (bottom) $\mathrm{QH}$ runs. The pink line represents the value of $\tilde{f}^{2}$. 

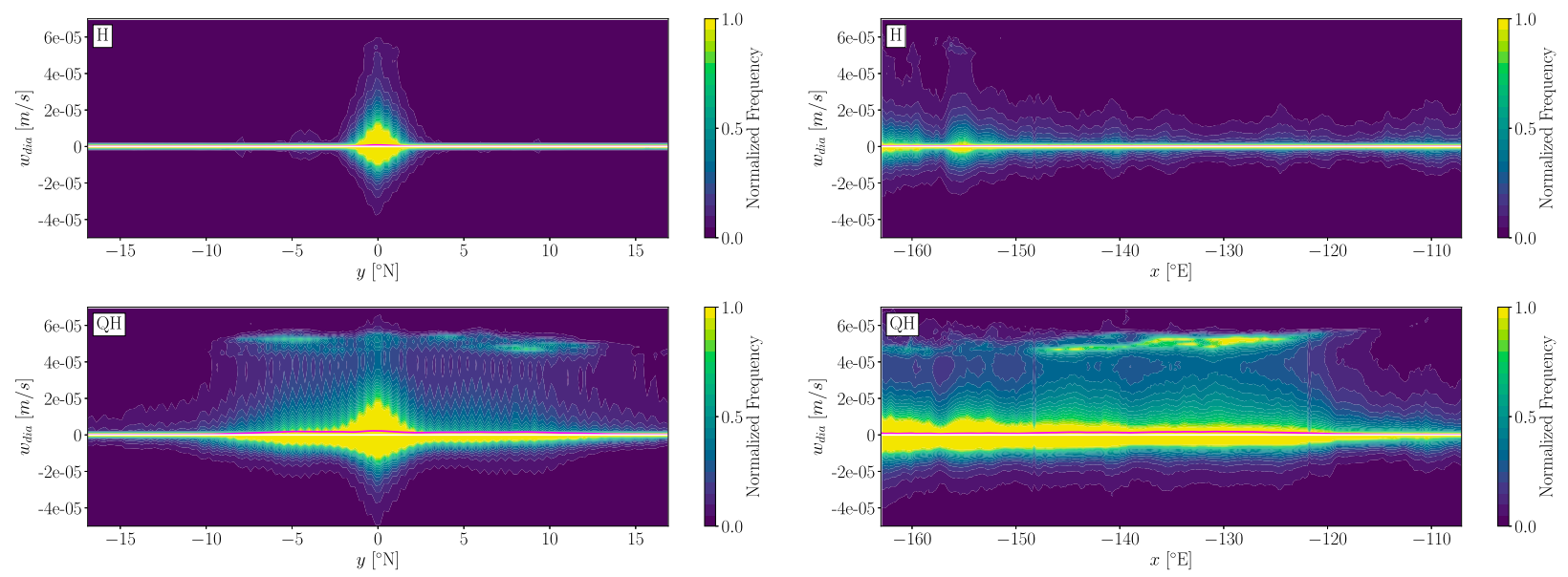

FIG. 19. Histogram of the diapycnal vertical velocity in the bottom $1000 \mathrm{~m}$ of the water column with the mean of the distribution (pink line) plotted as a function of (left) latitude and (right) longitude for the (top) $\mathrm{H}$ and (bottom) QH runs.

the abyss is crucial for the resulting upwelling, it is also necessary to investigate whether the feature shown in Fig. 2 is ubiquitous in the oceans. It would also be interesting to extend these results to other equatorial basins and provide a quantitative estimate of the potential contribution of the NT effects to closing deep cell of the MOC globally.

Finally, we hope that these results will motivate further observational studies in the abyssal equatorial ocean. To our knowledge, the only full-depth cross-equatorial microstructure profiles that have been made in the eastern equatorial Pacific were documented in Holmes et al. (2016), which are a main motivation for this study. We have investigated other microstructure profiles available in the Microstructure Database (https://microstructure.ucsd.edu/). Unfortunately, none of the profiles available in this database can be used to test the findings from this paper since the only profiles that were made over the full depth of the water column are either too far from the equator (i.e., more than $20^{\circ}$ away from the equator) or were obtained over rough topography. The only exception are the observations made in the Atlantic between $10^{\circ}$ and $25^{\circ} \mathrm{S}$ during the Brazil Basin Tracer Release Experiment. These results are described in Polzin et al. (1997) and point to weak mixing rates over smooth topography. We have estimated the abyssal stratification from the data obtained during this experiment and found that its values are much higher than the ones measured by Holmes et al. (2016) in the eastern Pacific, making $\Gamma \gg 1$ at the bottom of the ocean, which would imply that mixing due to NT effects would not be expected in this region. In addition, the equatorial wave field is weaker in the Atlantic versus the Pacific (Ascani et al. 2010). These facts all point to the Pacific being favored over the Atlantic for diapycnal upwelling in the deep branch of the MOC associated with NT effects. This hypothesis is consistent with the findings of Lumpkin and Speer (2007), that most of the abyssal return flow that closes the AMOC is occurring in the Pacific Ocean. Clearly, more observational efforts are needed to evaluate the mechanism described here in the abyssal equatorial ocean and the Eastern Pacific is the most relevant area of study for that.

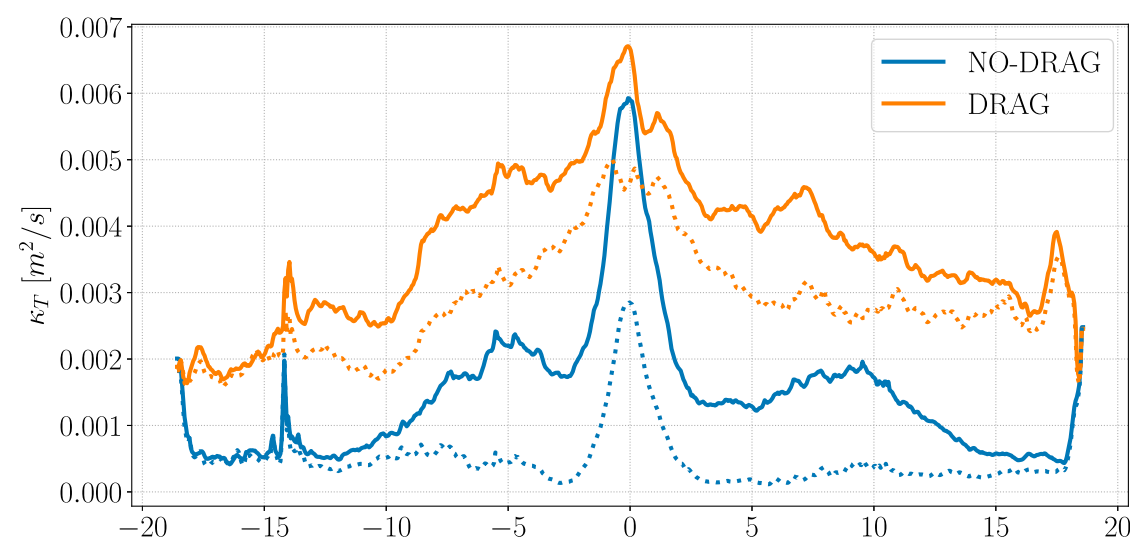

FIG. 20. The turbulent diffusivity averaged over the bottom $1000 \mathrm{~m}$ of the water column, over all longitudes and over the 5 years of simulation in the $\mathrm{H}$ (dotted lines) and QH (plain lines) runs with (orange) and without (blue) bottom drag. 
Acknowledgments. This work was a collaborative effort between scientists from France and the United States facilitated by a grant and a fellowship from the France-Stanford Center for Interdisciplinary Studies. It has also received support from a consortium of French research agencies, as part of CROCO's development project (GdR CROCO). Jonathan Gula was funded by the French National Agency for Research (ANR-19-CE01-0002-01). Jeroen Molemaker was funded by the Office of Naval Research (N00014-08-1-0597). Leif Thomas was funded by the Office of Naval Research (N00014-18-12798). B. Delorme gratefully acknowledges the hospitality of researchers, students, and staff at the Laboratoire d'Études en Géophysique et Océanographie Spatiales (LEGOS) and at the Laboratoire d'Océanographie Physique et Spatiale (LOPS) for hosting him over the course of this research project and making his stay very enjoyable and intellectually rewarding. Finally, we would like to acknowledge the two reviewers for their comments; we are particularly grateful to T. Durland for clarifying some subtleties of the spectral analysis of equatorial waves.

\section{APPENDIX}

\section{Quasi-Hydrostatic Version of CROCO (CROCO_QH)}

By default, CROCO solves the free-surface primitive equations using a robust and efficient time-splitting implementation (Shchepetkin and McWilliams 2005; Debreu et al. 2012). The primitive equations can be extended to form the quasihydrostatic $(\mathrm{QH})$ equations, relaxing the hypothesis of weak horizontal Coriolis force (White and Bromley 1995; Marshall et al.1997), thus adding a nonhydrostatic pressure component that is solved diagnostically.

In geophysical fluid dynamics, the traditional approximation (TA) takes the Coriolis force only partially into account by neglecting its components proportional to the cosine of latitude: $\tilde{f}=2 \Omega \cos \varphi$ [see Gerkema et al. (2008) for a review]. The justification for the TA lies in the hypothesis that the depth of the oceans is very thin compared to the radius of Earth. The vertical motions must then be much weaker than the horizontal ones, rendering the nontraditional (NT) Coriolis terms (i.e., associated with $\tilde{f}$ ) insignificant compared to the traditional terms (i.e., associated with $f$ ) and rendering the pressure field nearly hydrostatic. Similarly, strong vertical stratification in density, which suppresses vertical motions, also diminishes the role of NT terms. However, as shown in Delorme and Thomas (2019), this argument becomes weak near the equator since $\tilde{f} \gg f$, or in areas where the stratification is weak. In addition, the QH momentum equations are shown to be more dynamically consistent than the primitive hydrostatic equations and to correctly imply conservation laws for energy, angular momentum, and potential vorticity (White and Bromley 1995).

\section{a. Equations in Cartesian coordinate}

The momentum balance in the zonal and meridional directions $(x, y)$ is extended to include $\tilde{f}$ terms (zonal $u$ component):

$$
\frac{\partial u}{\partial t}=-\nabla \cdot(u \mathbf{v})+f v-\overbrace{\tilde{f} w}^{\mathrm{NT}_{x}}-\frac{\partial \phi}{\partial x}+\mathscr{F}_{u}+\mathscr{D}_{u}
$$

$$
\frac{\partial v}{\partial t}=-\nabla .(v \mathbf{v})-f u-\frac{\partial \phi}{\partial y}+\mathscr{F}_{v}+\mathscr{D}_{v} .
$$

The $\mathrm{QH}$ balance is used for the vertical momentum equation, where the zonal flow partially balances the pressure gradient:

$$
\frac{\partial \phi}{\partial z}=-\frac{\rho g}{\rho_{0}}+\overbrace{\tilde{f} u}^{\mathrm{NT}_{z}} .
$$

The variables used are

- $\mathscr{D}_{u}, \mathscr{D}_{v}$ : diffusive terms,

- $\mathscr{F}_{u}, \mathscr{F}_{v}$ : forcing terms,

- $f(x, y)$ : traditional Coriolis parameter $2 \Omega \sin \varphi$,

- $\tilde{f}(x, y)$ : nontraditional Coriolis parameter $2 \Omega \cos \varphi$,

- $g$ : acceleration of gravity,

- $\phi(x, y, z, t)$ : dynamic pressure $\phi=P / \rho_{0}$, with $P$ the total pressure,

- $\rho_{0}+\rho(x, y, z, t)$ : total in situ density, and

- $u, v, w$ : the $(x, y, z)$ components of vector velocity $\mathbf{v}$.

\section{b CROCO_QH implementation}

CROCO is implemented in a generalized stretched vertical coordinate system and orthogonal coordinate transformation in the horizontal that allows laterally variable grid resolution and orientation. In this case, NT terms for both horizontal momentum components are needed:

$$
\begin{aligned}
& \mathrm{NT}_{x}=-\tilde{f} w \cos \alpha \\
& \mathrm{NT}_{y}=+\tilde{f} w \sin \alpha
\end{aligned}
$$

where $\alpha$ is the angle between north and the $y$ grid axis. The nontraditional term $\mathrm{NT}_{z}=\tilde{f} u$ in the quasi-hydrostatic equation is introduced as a correction to density:

$$
\frac{\partial \phi}{\partial z}=-\frac{(\rho+\delta \rho)}{\rho_{0}} g
$$

with

$$
\delta \rho=-\frac{\rho_{0}}{g} \tilde{f}(u \cos \alpha-v \sin \alpha) .
$$

CROCO_QH has been validated on standard QH test cases using the full nonhydrostatic equations that had similar solutions. Obviously, the $\mathrm{QH}$ version is much faster than the fully nonhydrostatic model and, therefore, a better choice for our problem.

\section{REFERENCES}

Andersson, A., K. Fennig, C. Klepp, S. Bakan, H. Grassl, and J. Schulz, 2010: The Hamburg Ocean atmosphere parameters and fluxes from satellite data - HOAPS-3. Earth Syst. Sci. Data, 2, 215-234, https://doi.org/10.5194/essd-2-215-2010.

Ascani, F., E. Firing, P. Dutrieux, J. P. McCreary, and A. Ishida, 2010: Deep equatorial ocean circulation induced by a forced dissipated Yanai beam. J. Phys. Oceanogr., 40, 1118-1142, https://doi.org/10.1175/2010JPO4356.1.

Barnier, B., L. Siefried, and P. Marchesiello, 1995: Thermal forcing for a global ocean circulation model using a three-year 
climatology of ECMWF analyses. J. Mar. Syst., 6, 363-380, https://doi.org/10.1016/0924-7963(94)00034-9.

Becker, J. J., D. T. Sandwell, W. H. F. Smith, and J. Braud, 2009: Global bathymetry and elevation data at 30 arc seconds resolution: SRTM30 _ PLUS. Mar. Geod., 32, 355-371, https:// doi.org/10.1080/01490410903297766.

Beckmann, A., and D. B. Haidvogel, 1993: Numerical simulation of flow around a tall isolated seamount. Part I: Problem formulation and model accuracy. J. Phys. Oceanogr., 23, 1736-1753, https://doi.org/10.1175/1520-0485(1993)023<1736:NSOFAA > 2.0.CO;2.

Carton, J. A., and B. S. Giese, 2008: A reanalysis of ocean climate using Simple Ocean Data Assimilation (SODA). J. Phys. Oceanogr., 136, 2999-3017, https://doi.org/10.1175/2007MWR1978.1.

Conkright, M., R. A. Locarnini, H. Garcia, T. O’Brien, T. Boyer, C. Stephens, and J. Antonov, 2002: World Ocean Atlas 2001: Objective analyses, data statistics, and figures. National Oceanographic Data Center Internal Rep. 17, CD-ROM documentation, $17 \mathrm{pp}$.

Cox, M., 1980: Generation and propagation of 30-day waves in a numerical model of the Pacific.J. Phys. Oceanogr., 10, 1168-1186, https://doi.org/10.1175/1520-0485(1980)010<1168: GAPODW $>2.0 . \mathrm{CO} ; 2$.

Cravatte, S., E. Kestenare, F. Marin, P. Dutrieux, and E. Firing, 2017: Subthermocline and intermediate zonal currents in the tropical Pacific Ocean: Paths and vertical structure. J. Phys. Oceanogr., 47, 2305-2324, https://doi.org/10.1175/JPO-D-170043.1.

Debreu, L., P. Marchesiello, P. Penven, and G. Cambon, 2012: Two-way nesting in split-explicit ocean models: Algorithms, implementation and validation. Ocean Modell., 49-50, 1-21, https://doi.org/10.1016/j.ocemod.2012.03.003.

Delorme, B. L., and L. N. Thomas, 2019: Abyssal mixing through critical reflection of equatorially trapped waves off smooth topography. J. Phys. Oceanogr., 49, 519-542, https://doi.org/ 10.1175/JPO-D-18-0197.1.

Durland, T. S., and J. T. Farrar, 2012: The wavenumber-frequency content of resonantly excited equatorial waves. J. Phys. Oceanogr., 42, 1834-1858, https://doi.org/10.1175/JPO-D-110234.1.

Farrar, J. T., 2008: Observations of the dispersion characteristics and meridional sea level structure of equatorial waves in the Pacific Ocean. J. Phys. Oceanogr., 38, 1669-1689, https:// doi.org/10.1175/2007JPO3890.1.

— inertia-gravity and mixed Rossby-gravity waves in the equatorial Pacific Ocean. J. Phys. Oceanogr., 42, 1859-1881, https:// doi.org/10.1175/JPO-D-11-0235.1.

Ferrari, R., 2014: What goes down must come up. Nature, 513, 179180, https://doi.org/10.1038/513179a.

Gerkema, T., and V. I. Shrira, 2005: Near-inertial waves on the "nontraditional" $\beta$ plane. J. Geophys. Res., 110, C01003, https://doi.org/10.1029/2004JC002519.

— J. J. Zimmerman, L. R. Maas, and H. Van Haren, 2008: Geophysical and astrophysical fluid dynamics beyond the traditional approximation. Rev. Geophys., 46, RG2004, https:// doi.org/10.1029/2006RG000220.

Gula, J., J. M. Molemaker, and J. C. McWilliams, 2015: Gulf stream dynamics along the southeastern U. S. seaboard. J. Phys. Oceanogr., 45, 690-715, https://doi.org/10.1175/JPOD-14-0154.1.

Holmes, R. M., J. N. Moum, and L. N. Thomas, 2016: Evidence for seafloor-intensified mixing by surface-generated equatorial waves. Geophys. Res. Lett., 43, 1202-1210, https://doi.org/ 10.1002/2015GL066472.

Jullien, S., and Coauthors, 2019: CROCO'S technical and numerical documentation. Tech. Rep., CNRS-Ifremer-Inria-IRDSHOM, 142 pp.

Kelly, B. G., S. D. Meyers, and J. J. O'Brien, 1995: On a generating mechanism for Yanai waves and the 25-day oscillation. J. Geophys. Res., 100, 10 589-10 612, https://doi.org/10.1029/ 94JC02911.

Kessler, W. S., and J. P. McCreary, 1993: The annual wind-driven Rossby wave in the subthermocline equatorial Pacific. J. Phys. Oceanogr., 23, 1192-1207, https://doi.org/10.1175/15200485(1993)023<1192:TAWDRW>2.0.CO;2.

Kindle, J. C., and J. D. Thompson, 1989: The 26- and 50-day oscillations in the western Indian Ocean: Model results. J. Geophys. Res., 94, 4721-4736, https://doi.org/10.1029/JC094iC04p04721.

Large, G. S., and W. G. Yeager, 2009: The global climatology of an interannually varying air - Sea flux data set. Climate Dyn., 33, 341-364, https://doi.org/10.1007/s00382-008-0441-3.

Large, W. G., J. C. McWilliams, and S. C. Doney, 1994: Oceanic vertical mixing: A review and a model with a nonlocal boundary-layer parameterization. Rev. Geophys., 32, 363-403, https://doi.org/10.1029/94RG01872.

Lemarié, F., J. Kurian, A. F. Shchepetkin, M. Jeroen Molemaker, F. Colas, and J. C. McWilliams, 2012: Are there inescapable issues prohibiting the use of terrain-following coordinates in climate models? Ocean Modell., 42, 57-79, https://doi.org/ 10.1016/j.ocemod.2011.11.007.

Lumpkin, R., and K. Speer, 2007: Global ocean meridional overturning. J. Phys. Oceanogr., 37, 2550-2562, https://doi.org/ 10.1175/JPO3130.1.

Lyman, J. M., G. C. Johnson, and W. S. Kessler, 2007: Distinct 17and 33-day tropical instability waves in subsurface observations. J. Phys. Oceanogr., 37, 855-872, https://doi.org/10.1175/ JPO3023.1.

Malardé, J.-P., P. De Mey, C. Périgaud, and J.-F. Minster, 1987: Observation of long equatorial waves in the Pacific Ocean by Seasat altimetry. J. Phys. Oceanogr., 17, 2273-2279, https:// doi.org/10.1175/1520-0485(1987)017<2273:OOLEWI>2.0.CO;2.

Marchesiello, P., J. C. McWilliams, and A. Shchepetkin, 2001: Open boundary conditions for long-term integration of regional oceanic models. Ocean Modell., 3, 1-20, https://doi.org/ 10.1016/S1463-5003(00)00013-5.

Marshall, J., C. Hill, L. Perelman, and A. Adcroft, 1997: Hydrostatic, quasi-hydrostatic, and nonhydrostatic ocean modeling. J. Geophys. Res., 102, 5733-5752, https://doi.org/10.1029/96JC02776.

Mason, E., J. Molemaker, A. F. Shchepetkin, F. Colas, J. C. McWilliams, and P. Sangrà, 2010: Procedures for offline grid nesting in regional ocean models. Ocean Modell., 35, 1-15, https://doi.org/10.1016/j.ocemod.2010.05.007.

McPhaden, M. J., and R. A. Knox, 1979: Equatorial Kelvin and inertio-gravity waves in zonal shear flow. J. Phys. Oceanogr., 9, 263-277, https://doi.org/10.1175/1520-0485(1979)009<0263: EKAIGW $>2.0 . \mathrm{CO} ; 2$.

Ménesguen, C., A. Delpech, F. Marin, S. Cravatte, and R. Schopp, 2019: Observations and mechanisms for the formation of deep equatorial and tropical circulation. Earth Space Sci., 6, 370386, https://doi.org/10.1029/2018EA000438.

Molemaker, J. M., J. C. McWilliams, and W. K. Dewar, 2015: Submesoscale instability and generation of mesoscale anticyclones near a separation of the California undercurrent. J. Phys. Oceanogr., 45, 613-629, https://doi.org/10.1175/JPOD-13-0225.1. 
Penven, P., L. Debreu, P. Marchesiello, and J.C. McWilliams, 2006: Evaluation and application of the ROMS 1-way embedding procedure to the central California upwelling system. Ocean Modell., 12, 157-187, https://doi.org/10.1016/j.ocemod.2005.05.002.

Polzin, K., J. Toole, J. R. Ledwell, and R. W. Schmitt, 1997: Spatial variability of turbulent mixing in the abyssal ocean. Science, 276, 93-96, https://doi.org/10.1126/ science.276.5309.93.

Qiao, L., and R. H. Weisberg, 1995: Tropical instability wave kinematics: Observations from the tropical instability wave experiments. J. Geophys. Res., 100, 8677-8693, https://doi.org/ 10.1029/95JC00305.

Risien, C. M., and D. B. Chelton, 2008: A global climatology of surface wind and wind stress fields from eight years of QuikSCAT scatterometer data. J. Phys. Oceanogr., 38, 23792413, https://doi.org/10.1175/2008JPO3881.1.

Shchepetkin, A. F., and J. C. McWilliams, 2005: The Regional Oceanic Modeling System (ROMS): A split-explicit, freesurface, topography-following-coordinate oceanic model. Ocean Modell., 9, 347-404, https://doi.org/10.1016/j.ocemod.2004.08.002.

__, and ——, 2009a: Computational Kernel algorithms for fine-scale, multiprocess, longtime oceanic simulations. Handb. Numer. Anal., 14, 121-183, https://doi.org/10.1016/ S1570-8659(08)01202-0.

_ and $\longrightarrow$, 2009b: Correction and commentary for "Ocean forecasting in terrain-following coordinates: Formulation and skill assessment of the regional ocean modeling system."
J. Comput. Phys., 228, 8985-9000, https://doi.org/10.1016/ j.jcp.2009.09.002.

— with a practical, "stiffened" equation of state. Ocean Modell., 38, 41-70, https://doi.org/10.1016/j.ocemod.2011.01.010.

Shinoda, T., 2012: Observation of first and second baroclinic mode Yanai waves in the ocean. Quart. J. Roy. Meteor. Soc., 138, 1018-1024, https://doi.org/10.1002/qj.968.

Smith, W. H. F., and D. T. Sandwell, 1997: Global sea floor topography from satellite altimetry and ship depth soundings. Science, 277, 1956-1962, https://doi.org/10.1126/SCIENCE.277.5334.1956.

White, A. A., and R. A. Bromley, 1995: Dynamically consistent, quasi-hydrostatic equations for global models with a complete representation of the Coriolis force. Quart. J. Roy. Meteor. Soc., 121, 399-418, https://doi.org/10.1002/qj.49712152208.

Winters, K. B., P. Bouruet-Aubertot, and T. Gerkema, 2011: Critical reflection and abyssal trapping of near-inertial waves on a beta-plane. J. Fluid Mech., 684, 111-136, https://doi.org/ 10.1017/jfm.2011.280.

Woodberry, E., M. E. Luther, and J. O. Brien, 1989: The winddriven seasonal circulation in the southern tropical Indian Ocean. J. Geophys. Res., 94, 17 985-18002, https://doi.org/ 10.1029/JC094iC12p17985.

Wunsch, C., and A. E. Gill, 1976: Observations of equatorially trapped waves in Pacific Sea level variations. Deep. Res. Oceanogr. Abstr., 23, 371-390, https://doi.org/10.1016/00117471(76)90835-4. 